\title{
Multidimensional solitons: Well-established results and novel findings
}

\author{
Boris A. Malomed \\ Department of Physical Electronics, School of Electrical Engineering, \\ Faculty of Engineering, Tel Aviv University, Tel Aviv 69978, Israel
}

\begin{abstract}
A brief review is given of some well-known and some very recent results obtained in studies of two- and three-dimensional (2D and 3D) solitons. Both zero-vorticity (fundamental) solitons and ones carrying vorticity $S=1$ are considered. Physical realizations of multidimensional solitons in atomic Bose-Einstein condensates (BECs) and nonlinear optics are briefly discussed too. Unlike 1D solitons, which are typically stable, 2D and 3D ones are vulnerable to the instability induced by the occurrence of the critical and supercritical collapse, respectively, in the same 2D and 3D models that give rise to the solitons. Vortex solitons are subject to a still stronger splitting instability. For this reason, a central problem is looking for physical settings in which $2 \mathrm{D}$ and $3 \mathrm{D}$ solitons may be stabilized. The review addresses one well-established topic, viz., the stabilization of the 3D and 2D states, with $S=0$ and 1, trapped in harmonic-oscillator (HO) potentials, and another topic which was developed very recently: the stabilization of $2 \mathrm{D}$ and $3 \mathrm{D}$ free-space solitons, which mix components with $S=0$ and \pm 1 (semi-vortices and mixed modes), in a binary system with the spinorbit coupling (SOC) between its components. In both cases, the generic situations are drastically different in the 2D and 3D geometries. In the former case, the stabilization mechanism creates a stable ground state (GS, which was absent without it), whose norm falls below the threshold value at which the critical collapse sets in. In the 3D geometry, the supercritical collapse does not allow to create a GS, but metastable solitons can be constructed.
\end{abstract}

List of acronyms: GPE - Gross-Pitaevskii equation; GS - ground state; HO - harmonic-oscillator (potential); MM - mixed mode; NLSE - nonlinear Schrödinger equation; SOC - spin-orbit coupling; SV - semi-vortex; VA variational approximation; VK - Vakhitov-Kolokolov (stability criterion)

\section{INTRODUCTION}

Self-trapping of multidimensional (two- and three-dimensional, 2D and 3D) localized modes in nonlinear dispersive/diffractive media, which are usually categorized as solitons, has been a topic of great interest in many areas of physics, as can be seen from reviews [1]-[3]. In particular, this area of the theoretical and experimental studies is highly relevant to nonlinear optics, as well as to nonlinear dynamics of matter waves in Bose-Einstein condensates (BECs). Other essential realizations of multidimensional solitons are known in models of ferromagnets [4], superconductors [5], and semiconductors [6], as well as in nuclear physics [7], the classical-field theory [8, 9] (including famous skyrmions [10], which may be considered as 3D solitons), and other fields.

In addition to the their significance to fundamental studies, 2D and 3D solitons offer potential applications. In particular, spatiotemporal optical solitons, alias "light bullets" 11], may be used as data carriers in all-optical dataprocessing schemes [12], and 3D matter-wave solitons are expected to provide a basis for highly precise interferometry [13].

Unlike 1D solitons, which are usually stable objects [2, 14], stability is a major issue for their 2D and 3D counterparts. Indeed, the most common cubic self-focusing nonlinearity, which can easily support 2D and 3D formal solitons solutions, gives rise to the wave collapse (critical collapse in 2D and supercritical collapse in 3D) [15], which completely destabilizes the respective soliton families. In particular, the first ever example of solitons which was introduced theoretically in nonlinear optics, viz., the Townes solitons [16], i.e., 2D self-trapped modes supported by the cubic selffocusing nonlinearity (in fact, these solutions were discovered before term "soliton" had been coined) are subject to the instability caused by the critical collapse, therefore they have never been observed in the experiment. Multidimensional solitons with embedded vorticity, alias $2 \mathrm{D}$ vortex rings and $3 \mathrm{D}$ vortex tori, which also exist in cubic media (in particular, there are higher-order versions of Townes solitons with embedded vorticity [17]), are vulnerable to a still stronger splitting instability initiated by modulational perturbations in the azimuthal direction; usually, the modulational instability splits the vortex ring into a few fragments, which resemble fundamental (zero-vorticity) solitons and suffer the destruction via the intrinsic collapse or decay into small-amplitude waves ("radiation") [1].

Thus, the stabilization of multidimensional fundamental and vortex solitons in physically relevant models is an issue of great significance to theoretical studies, and creation of the solitons in the respective physical settings is a challenge to the experiment. This paper offers a short review of the broad area of theoretical investigations of $2 \mathrm{D}$ and 3D solitons, selecting two topics for a relatively detailed presentation, one well-established, and one very recent. The former one, considered in Section II, relies upon the stabilization of both fundamental (zero-vorticity) solitons and 
ones with embedded vorticity $S=1$ by an external harmonic-oscillator (HO) trapping potential, axially or spherically symmetric one. This topic has been elaborated in detail in the course of the past 20 years [18 22]. A recently developed theme, presented in Section III, is the creation of stable solitons in the 2D and 3D free space (without the help from any external potential), which mix zero-vorticity and vortical components, by means of linear terms that account for the spin-orbit coupling (SOC) in binary BEC. This possibility for 2D and 3D solitons was discovered in Refs. [23] and 24], respectively.

Any stabilization mechanism must secure the solitons against the critical collapse in $2 \mathrm{D}$ settings, and against the supercritical collapse in the 3D geometry. This objective is achieved differently in 2D and 3D models, as explained below. In the former case, the stabilizing factor breaks the specific scale invariance, which underlies the critical collapse, and transforms any originally unstable fundamental soliton in the single-component model, or a mixed (fundamental-vortical) soliton in the binary SOC system, into a ground state (GS), which does not exists in the unstabilized 2D model. This is possible because the critical collapse sets in when the norm of the mode exceeds a certain threshold value, and the solitons get stabilized by pushing their norm below the collapse threshold. Vortex solitons with $S=1$ in the $2 \mathrm{D}$ single-component model may be stabilized too, as excited states, rather than the GS, provided that their norms are not too large either. On the other hand, in the 3D setting the threshold for the onset of the supercritical collapse is absent, hence a true GS cannot be created. Nevertheless, the stabilization of 3D fundamental solitons and vortical ones with $S=1$ in the single-component model, and of 3D mixed modes in the SOC system, is possible in the form of metastable states, which are robust against small perturbations.

\section{MULTIDIMENSIONAL SOLITONS IN TRAPPING POTENTIALS}

\section{A. The basic model}

A generic method for the stabilization of both fundamental and vortical solitons is the use of trapping potentials, the corresponding model being based on the following nonlinear Schrödinger equation (NLSE), alias the Gross-Pitaevskii equation (GPE) [25], for the atomic mean-field wave function $\psi(\mathbf{r}, t)$, where $\mathbf{r}=(x, y, z)($ or $\mathbf{r}=(x, y)$ in the $2 \mathrm{D}$ geometry) and $t$ are appropriately scaled spatial coordinates and time:

$$
i \frac{\partial \psi}{\partial t}=-\frac{1}{2} \nabla^{2} \psi+U(\mathbf{r}) \psi-|\psi|^{2} \psi
$$

Here, $\nabla^{2}$ is the 3D or 2D Laplacian, $U(\mathbf{r})$ is the trapping potential, and the negative sign in front of the cubic term implies that the nonlinearity is self-attractive. The trapping potential in the 3D setting may be quasi-two-dimensional, i.e., $U=U(x, y)[26]$.

In the application to optics, the evolution variable in Eq. (11) is replaced by the propagation distance ( $z$ ), while the longitudinal coordinate is replaced by the local time, $\tau \equiv t-z / V_{\mathrm{gr}}$, where $V_{\mathrm{gr}}$ is the group velocity of the electromagnetic carrier waves 2]. In this case, the trapping potential, which defines a waveguiding channel in the bulk medium by means of the local modulation of the refractive index, $\delta n=-U(x, y)$, may be only quasi-twodimensional, as it is virtually important to impose modulation of the refractive index providing the trapping in the temporal direction. The 2D version of Eq. (1), with the propagation distance $z$ replacing $t$, models the evolution of spatial light beams in the bulk medium with transverse coordinates $(x, y)$.

Axially symmetric potentials (including spherically isotropic ones), $U=U(\rho, z)$, where $(\rho, z, \theta)$ is the set of cylindrical coordinates, admit looking for solutions to Eq. (1) in the form of vortex modes,

$$
\psi=\exp (-i \mu t+i S \theta) R(\rho, z)
$$

with real chemical potential $\mu$ and integer vorticity $S$. Real amplitude function $R(\rho, z)$ satisfies the stationary equation,

$$
\mu u=-\frac{1}{2}\left(\frac{\partial^{2}}{\partial \rho^{2}}+\frac{1}{\rho} \frac{\partial}{\partial \rho}+\frac{\partial^{2}}{\partial z^{2}}-\frac{S^{2}}{\rho^{2}}\right) R+U(\rho, z) R-R^{3}
$$

supplemented by the boundary conditions (b.c.), $R(\rho, z) \rightarrow 0$ at $\rho \rightarrow \infty$ and $|z| \rightarrow \infty$, and $R \sim \rho^{S}$ at $\rho \rightarrow 0$ (assuming $S \geq 0)$.

Relevant to the experiments with BEC is the HO trapping potential [25],

$$
U(x, y, z)=\frac{1}{2}\left(x^{2}+y^{2}+\Omega^{2} z^{2}\right),
$$


where $\Omega^{2}$ accounts for the anisotropy of the trap, determining the aspect ratio, $\sqrt{\Omega}$, between trapping lengths in plane $(x, y)$ and along axis $z$. Accordingly, the cases of $\Omega^{2} \gg 1$ and $\Omega^{2} \ll 1$ corresponding, respectively, to nearly two-dimensional ("pancake-shaped", see Ref. [27] and references therein) and nearly one-dimensional ("cigar-shaped" [28]) condensates. Equation (11) conserves the energy,

$$
E=\frac{1}{2} \iiint\left[\left(\left|\frac{\partial \psi}{\partial x}\right|^{2}+\left|\frac{\partial \psi}{\partial y}\right|^{2}+\left|\frac{\partial \psi}{\partial z}\right|^{2}\right)+\left(x^{2}+y^{2}+\Omega^{2} z^{2}\right)|\psi|^{2}-|\psi|^{4}\right] d x d y d z
$$

norm (scaled number of atoms in the BEC), or the total energy in optics),

$$
N=\iiint|\psi(x, y, z)|^{2} d x d y d z
$$

and $z$-component of the angular momentum,

$$
M_{z}=i \iiint\left(y \frac{\partial \psi}{\partial x}-x \frac{\partial \psi}{\partial y}\right) \psi^{*} d x d y d z
$$

with $*$ standing for the complex conjugate (in the case of the isotropic trap, $\Omega=1$, all the three components of the angular momentum are conserved). For the stationary state (2) with a definite value of $S$, relation $M_{z}=S N$ holds.

In this section, the basic results for the structure and stability of trapped solitons and solitary vortices are summarized for the HO potential (44), following, chiefly, Refs. 21] and [22]. On the other hand, spatially periodic (lattice) potentials also offer an efficient tool for the stabilization of $2 \mathrm{D}$ and $3 \mathrm{D}$ fundamental and vortex solitons, as was predicted in various settings [26, 29], and demonstrated experimentally for 2D optical solitons with embedded vorticity in photonic lattices [30], and for 2D plasmon-polariton solitons in microcavities with a lattice structure [31].

\section{B. Stationary 3D modes: analytical and numerical results}

Before proceeding to the presentation of numerical findings, it is relevant to display approximate analytical results which are available in the present setting. In the linear limit, Eq. (1) is tantamount to the quantum-mechanical Schrödinger equation for the 3D anisotropic HO. In the Cartesian coordinates, the corresponding eigenfunctions are built as

$$
\psi_{j k l}(x, y, z, t)=e^{-i \mu_{0} t} \Phi_{j}(x) \Phi_{k}(y) \Phi_{l}(\sqrt{\Omega} z)
$$

where $\Phi_{j}, \Phi_{k}$ and $\Phi_{l}$ are stationary wave functions of $1 \mathrm{D}$ harmonic oscillators with quantum numbers $j, k, l$, which correspond to energy eigenvalues $j+1 / 2, k+1 / 2$ and $(l+1 / 2) \Omega$, respectively, the full chemical potential being

$$
\mu_{0}=j+k+1+(l+1 / 2) \Omega .
$$

The states which go over into ones (2) with vorticity $S$ in the nonlinear model are constructed as combinations of factorized wave functions (8) with $l=0$ and $j+k=S$. Since the correction to $\mu$ from the self-attractive nonlinearity is negative, this restriction and Eq. (9) with $l=0$ impose a bound on $\mu$,

$$
\mu \leq \mu_{0}=S+1+(1 / 2) \Omega
$$

In particular, the eigenfunctions of the linear model, with vorticities $S=1$ and $S=2$, are

$$
\begin{gathered}
\psi_{\text {linear }}^{(S=1)}=\psi_{100}+i \psi_{010} \equiv \rho \exp \left[-(2+\Omega / 2) i t+i \theta-\left(\rho^{2}+z^{2}\right) / 2\right], \\
\psi_{\text {linear }}^{(S=2)}=\psi_{200}-\psi_{020}+2 i \psi_{110} \equiv \rho^{2} \exp \left[-(3+\Omega / 2) i t+2 i \theta-\left(\rho^{2}+z^{2}\right) / 2\right] .
\end{gathered}
$$

To apply the variational approximation (VA) [32] to the nonlinear model, note that Eq. (3) can be derived from Lagrangian

$$
L=\int_{0}^{\infty} \rho d \rho \int_{0}^{+\infty} d z\left[\left(\frac{\partial R}{\partial z}\right)^{2}+\left(\frac{\partial R}{\partial \rho}\right)^{2}+\left(\frac{S}{\rho}\right)^{2} R^{2}-2 \mu R^{2}+\left(\rho^{2}+\Omega^{2} z^{2}\right) R^{2}-R^{4}\right]
$$


(a)

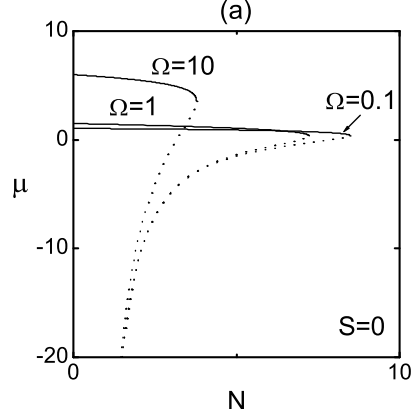

(b)

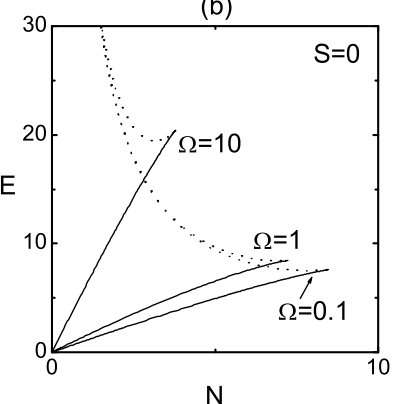

FIG. 1: (a) The chemical potential and (b) energy, defined as per Eq. (5), versus norm $N$ for the fundamental $(S=0)$ modes trapped in anisotropic HO potential (4) with $\Omega=10,1$, and 0.1, as found from numerical solution of Eq. (3). Solid and dotted portions of the curves denote, respectively, stable and unstable parts of the solution families, as identified by a numerical solution of the eigenvalue problem based on Bogoliubov - de Gennes equations (16). See further details in Ref. [22].

A natural ansatz for the trapped vortices is

$$
R(\rho, z)=A \rho_{0}^{S} \exp \left[-\rho^{2} /\left(2 \rho_{0}^{2}\right)-z^{2} /\left(2 z_{0}^{2}\right)\right],
$$

where amplitude $A$, as well as the radial and vertical widths, $\rho_{0}$ and $z_{0}$, are free parameters, norm (6) of this ansatz being $N=\left(\pi^{3 / 2} S !\right) M, M \equiv A^{2} \rho_{0}^{4} h_{0}$ (below, $M$ replaces $A$ as one of variational parameters). The trapped modes with $S \geq 1$ are shaped as vortex tori ("doughnuts"), see a typical example below in Fig. 4 . This shape is (qualitatively) correctly captured by ansatz (14).

Further results produced by the VA are given here for $S=0$ and 1 , as all vortices with $S \geq 2$ are unstable, see below. The substitution of ansatz (14) with $S=1$ in Lagrangian (13) yields $L=$ $(\sqrt{\pi} / 8) M\left[-4 \mu+z_{0}^{-2}+4 \rho_{0}^{-2}+4 \rho_{0}^{2}+\Omega^{2} z_{0}^{2}-M\left(2 \sqrt{2} \rho_{0}^{2} z_{0}\right)^{-1}\right]$. The first variational equation, $\partial L / \partial \rho_{0}=0$, yields $M=8 \sqrt{2}\left(1-\rho_{0}^{4}\right) h_{0}$, which predicts that the vortex may only exist if it is narrow enough in the radial direction, $\rho_{0}<$ 1. Two other equations, $\partial L / \partial z_{0}=\partial L / \partial M=0$, yield $z_{0}^{2}\left[1-2(1-\mu) \rho_{0}^{2}-3 \rho_{0}^{4}\right]=2 \rho_{0}^{2}, \Omega^{2} \rho_{0}^{2} z_{0}^{4}-2 \rho_{0}^{4} z_{0}^{2}-\rho_{0}^{2}+2 z_{0}^{2}=0$.

Numerical solution of the variational equations generates $\mu(N)$ dependences for families of the fundamental $(S=0)$ and vortical $(S=1)$ trapped modes which are displayed, severally, in Figs. 1(a) and 2 (a) for $\Omega=10,1$, and 0.1 , which correspond to the pancake-like, isotropic, and cigar-shaped configurations, respectively, while the corresponding $E(N)$ curves are displayed in Figs 1(b) and 2(b). The same dependences, obtained from numerical solution of stationary equation (3), are displayed too, demonstrating the accuracy of the VA. It is seen that the properties of the modes trapped in the isotropic $(\Omega=1)$ and cigar-shaped $(\Omega=0.1)$ HO potentials are quite close, being strongly different from those is the pancake trap $(\Omega=10)$. Another obvious feature of the figure is the presence of a largest norm, which corresponds to the turning point of the $\mu(N)$ curve and bounds the existence of the trapped modes. This limitation is caused by the presence of the supercritical collapse in the 3D model [15]: if the norm is too large, the trend to the collapse, driven by the cubic self-attraction, cannot be balanced by the quantum pressure (the gradient part of energy (50). The largest norm in this model was numerically found, as a function $\Omega$, in Ref. 19]. Another implication of the possibility of the supercritical collapse is that all the stable modes found in the 3D model are actually metastable ones. They cannot play the role of the GS, which does not exist in the model admitting the supercritical collapse.

\section{Stability of trapped 3D fundamental and vortex modes}

The stability of stationary solutions produced by Eq. (3) is identified through the computation of (generally, complex) eigenvalues $\lambda$ of small perturbations. To this end, a perturbed solution to Eq. (10) is looked for as

$$
\psi(x, y, z, t)=\left[R(\rho, z)+u(\rho, z) \exp (\lambda t+i L \theta)+v^{*}(\rho, z) \exp \left(\lambda^{*} t-i L \theta\right)\right] \exp (i S \theta-i \mu t),
$$


(a)

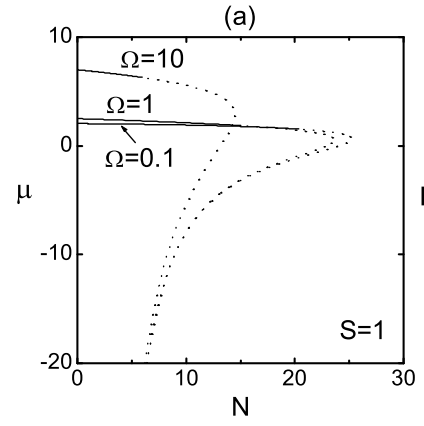

(b)

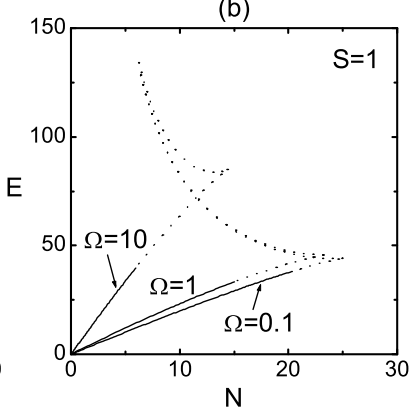

FIG. 2: The same as in Fig. 1 but for trapped vortex modes with $S=1$.

where $(u, v)$ are eigenmodes of infinitesimal perturbations corresponding to integer values of azimuthal index $L$. The substitution of ansatz (15) in Eq. (11) and linearization lead to the Bogoliubov - de Gennes equations [25],

$$
\begin{gathered}
(i \lambda+\mu) u+\frac{1}{2}\left[\frac{\partial^{2}}{\partial \rho^{2}}+\frac{1}{\rho} \frac{\partial}{\partial \rho}+\frac{\partial^{2}}{\partial z^{2}}-\frac{(S+L)^{2}}{\rho^{2}} u-\rho^{2}\right] u+R^{2}(v+2 u)=0 \\
(-i \lambda+\mu) v+\frac{1}{2}\left[\frac{\partial^{2}}{\partial \rho^{2}}+\frac{1}{\rho} \frac{\partial}{\partial \rho}+\frac{\partial^{2}}{\partial z^{2}}-\frac{(S-L)^{2}}{\rho^{2}} v-\rho^{2}\right] v+R^{2}(u+2 v)=0
\end{gathered}
$$

supplemented by the boundary conditions demanding that $u(\rho, z)$ and $v(\rho, z)$ decay exponentially at $\rho \rightarrow \infty$ and $|z| \rightarrow \infty$, and decay as $\rho^{|S \pm L|}$ at $\rho \rightarrow 0$. The stability of the underlying stationary mode is secured by the condition that all eigenvalues $\lambda$ produced by numerical solution of Eq. [16) [20] must be pure imaginary. The findings are included in Figs. 1 and 2 where stable and unstable portions of the solution families are distinguished. The results of the stability analysis are collected in the diagram which displays stability regions in the plane of $(\mu, \Omega)$ in Fig. [3]

The stability of the fundamental trapped states $(S=0)$ precisely complies with the Vakhitov-Kolokolov (VK) criterion [15, 33], $d \mu / d N \leq 0$, which is a necessary but, in the general case, not sufficient stability condition. On the other hand, the stability region of the vortex mode with $S=1$ is essentially smaller than formally predicted by this criterion. In the region with the VK criterion still holds for the vortices, but they are actually unstable, they are vulnerable to the above-mentioned splitting instability induced by perturbations with $L=2$ and 3 in Eq. (15). The vortex modes with $S \geq 2$ were found to be completely unstable.

A noteworthy feature revealed by Figs. 1 and 2 is that the smallest and largest maximum values of $N$, up to which the trapped modes with $S=0$ or $S=1$ remain stable, are achieved, respectively, at the largest and smallest values of $\Omega$. In particular, the quasi-1D "tubular" vortical modes with $S=1$ [34], which are generated by the model at $\Omega \ll 1$, are tightly confined in the corresponding cigar-shaped trap, that suppresses their destabilization by the splitting.

As said above, at $\Omega \rightarrow \infty$ the 3D setting shrinks into its $2 \mathrm{D}$ counterpart, where trapped modes with $S=1$ have a finite stability region, $\mu_{0}-\mu_{\mathrm{cr}} \approx 0.724$, see below. Therefore, taking into regard that Eq. (10) yields $\mu_{0}(\Omega)=2+\Omega / 2$ for $S=1$, the lower stability border $\left(\mu=\mu_{\mathrm{cr}}\right)$ for $S=1$ in Fig. 3 becomes, at large $\Omega$, asymptotically linear and parallel to the upper existence border, $\mu_{\mathrm{cr}} \approx \mu_{0}(\Omega)-0.724=1.276+\Omega / 2$. On the other hand, the stability region for the fundamental trapped modes expands at $\Omega \rightarrow \infty$, because the entire fundamental family is stable in the $2 \mathrm{D}$ limit, see below too.

The predictions for the stability based on the computation of the stability eigenvalues are corroborated by direct simulations of Eq. (1), starting with stationary modes to which small arbitrary perturbations are added. The robustness of stable vortices is illustrated by Fig. [4, which demonstrates that they absorb the perturbations and clean up themselves.

Lastly, it is relevant to stress that stable matter-wave solitons, created experimentally in condensates of ${ }^{7} \mathrm{Li} 28$ and ${ }^{85} \mathrm{Rb}[35]$ atoms loaded in cigar-shaped traps, may be well described by the above solutions for $\Omega \simeq 0.1-0.05$. On the other hand, stable solitons were also observed in the post-collapse state of the ${ }^{85} \mathrm{Rb}$ condensate trapped in the HO potential with $\Omega \approx 0.4$, which is much closer to the isotropic configuration. 


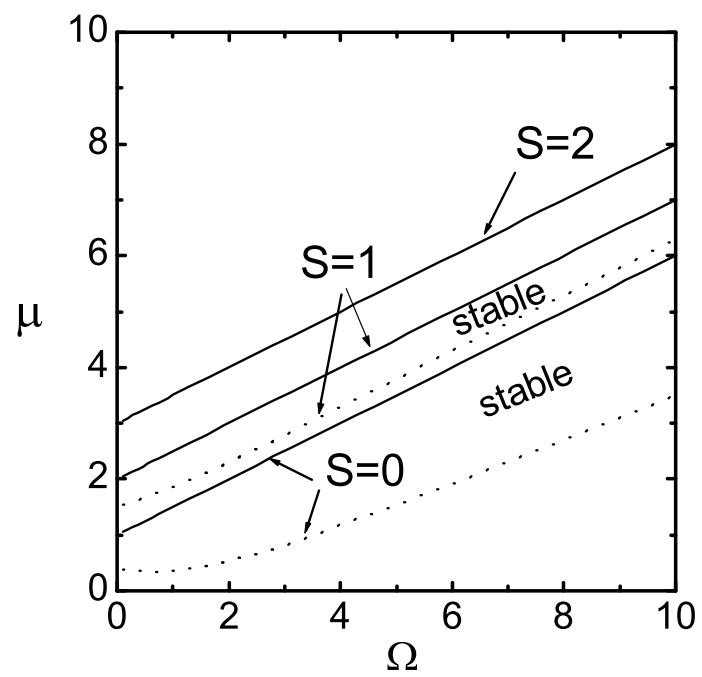

FIG. 3: Stability regions for the 3D trapped states with vorticities $S=0,1$, and 2, as per Ref. [22]. The solutions exist and are stable between the upper border (solid line), which is given by $\mu=\mu_{0}$ in Eq. (10), and the numerically found lower stability border (dotted curve). Vortices with $S=2$ have no stability domain.

(a)

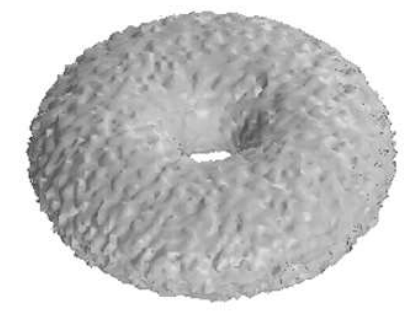

(b)

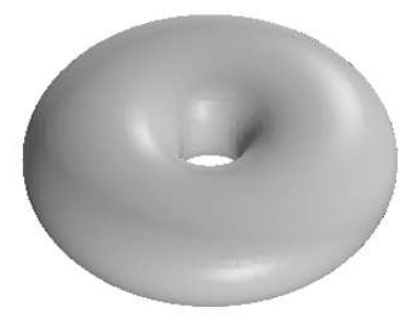

FIG. 4: Self-cleaning of a stable 3D vortex torus with $S=1$ in the isotropic model $(\Omega=1)$ after the application of a random perturbation at the amplitude level of $10 \%$, as per Ref. [22]. Panels (a) and (b) display. severally, the initial shape of the vortex at $t=0$, and the shape at $t=120$. The unperturbed vortex has chemical potential $\mu=2$ and norm $N=12.55$.

\section{2D fundamental and vortex trapped modes}

As said above, taking the limit of $\Omega \rightarrow \infty$ in HO potential (4) leads to the shrinkage of the 3D model into its 2D simplification, with Eqs. (GPE), (3), (5), (6), (7), and (16) carrying over into their 2D counterparts, and the 2D HO potential taking the form of $U(x, y)=(1 / 2)\left(x^{2}+y^{2}\right)$. In particular, stationary solutions of the 2D version of Eq. (11) are looked for as $\psi=\exp (-i \mu t+i S \theta) R(\rho)$, cf. Eq. (2), where $\mu$ is realized as the chemical potential of the 2D model, i.e., its limit form corresponding to the linear equation is

$$
\mu_{0}^{(2 \mathrm{D})}=S+1,
$$




\section{cf. Eq. (10).}

The shape and stability of the 2D trapped modes was studied in a number of works [18, 21]. The results, produced by the computation of the stability eigenvalues in the framework of the 2D version of Eq. (16), are summarized in Fig. 5. which demonstrates that the family of the fundamental $(S=0)$ trapped modes is entirely stable in its existence region,

$$
0 \leq N<N_{\max }^{(S=0)} \approx 5.85
$$

where $N_{\max }^{(S=0)}$ is the numerically found value of the norm of the Townes soliton, which determines the threshold for the onset of the critical collapse in the 2D NLSE [15] (the variational method makes it possible to produce an analytical approximation, $N_{\max }^{(S=0)} \approx 2 \pi$, with a relative error $\approx 7 \%$ 37]). In the free space (in the absence of any trapping potential), the family of the Townes solitons is degenerate, in the sense that they all have the single value of the norm, which is exactly equal to $N_{\max }^{(S=0)}$, independently of the soliton's chemical potential, $\mu$. The degeneracy is a consequence of the specific scaling invariance of the NLSE in 2D [15]. The trapping potential introduces a characteristic spatial scale (the standard HO length, in dimensional units, or merely the spatial period, in the case of lattice potentials), which breaks the scaling invariance and thus lifts the degeneracy, making $N$ a function of $\mu$. In fact, Eq. (18) demonstrates that the degeneracy is lifted so that the soliton's norm falls below the collapse-onset threshold. This circumstance lends the trapped modes with $S=0$ protection against the collapse, i.e.,, stability, and actually makes them the system's GS, which did not exist in the absence of the trapping potential. This is a major difference from the stabilization mechanism provided by the trapping potential in 3D, where, as said above, the GS cannot exist (as the supercritical collapse is possible at any value of the norm), and only metastability of the trapped modes is possible.

The family of Townes solitons with embedded vorticity $S=1$ is degenerate too in the free space, being pinned to the single value of the norm, $N_{\max }^{(S=1)} \approx 24.1$ [17]. As seen in Fig. 5] the HO trapping potential stabilizes them in interval

$$
0 \leq N<7.79 \approx 0.32 N_{\max }^{(S=1)}
$$

the corresponding stability interval in terms of the chemical potential being $1.27575 \equiv \mu_{\mathrm{cr}}<\mu \equiv \mu_{0}^{(2 \mathrm{D})}(S=1) \equiv 2$ (the right edge of the interval is determined by Eq. (17)). As for the vortices with $S \geq 2$, they remain completely unstable, as in the 3D model.

The stability of the trapped vortex modes with $S=1$, predicted through the computation of the corresponding eigenvalues, was corroborated by direct simulations of the perturbed evolution, see a typical example in Fig. 6

The direct simulations also reveal an interesting dynamical regime of the trapped vortices with $S=1$ in interval

$$
0.32 N_{\max }^{(S=1)} \approx 7.79<N<10.30 \approx 0.43 N_{\max }^{(S=1)}
$$

adjacent to one given by Eq. (19). In this interval, the evolution of the unstable vortex is regular (time-periodic), as shown in Fig. 7, it spits into two fragments which then recombine back into the vortex. The splitting-recombination cycles recur periodically, keeping the vorticity of the configuration. In this dynamical regime, the trapped vortices may be considered as semi-stable modes.

The vortices with still larger values of the norm, $N>10.30$, also split into two fragments, which, however, fail to recombine. Instead, each one quickly blows up, i.e., collapses. Note that the corresponding effective collapse threshold, $N \approx 10.30$, is close to the double collapse threshold of the fundamental solitons, $2 N_{\max }^{(S=0)} \approx 11.70$, which is consistent with the observation that the two fragments tend to suffer the intrinsic collapse if they do not recombine.

A noteworthy generalization of the above analysis was performed for a system of two nonlinearily coupled fields, which may be realized as a binary BEC, or as the copropagation of two optical beams in a bulk waveguide 38. The respective coupled $2 \mathrm{D}$ GPE/NLSE system is

$$
\begin{aligned}
& i \frac{\partial \psi_{1}}{\partial t}=\left[-\frac{1}{2} \nabla^{2}+\frac{1}{2}\left(x^{2}+y^{2}\right)-\left(\left|\psi_{1}\right|^{2}+\eta\left|\psi_{2}\right|^{2}\right)\right] \psi_{1}, \\
& i \frac{\partial \psi_{2}}{\partial t}=\left[-\frac{1}{2} \nabla^{2}+\frac{1}{2}\left(x^{2}+y^{2}\right)-\left(\left|\psi_{2}\right|^{2}+\eta\left|\psi_{1}\right|^{2}\right)\right] \psi_{2},
\end{aligned}
$$

where $\eta$ is the relative strength of the attraction $(\eta>0)$ or repulsion $(\eta<0)$ between the components. This system gives rise to composite modes with hidden vorticity, formed by the components with equal norms $N / 2$ and opposite vorticities, $S_{1,2}= \pm 1$. Such modes are stable, roughly, in the parameter region with $0 \leq N<8,-1.0 \leq \beta \leq 0.2$. 


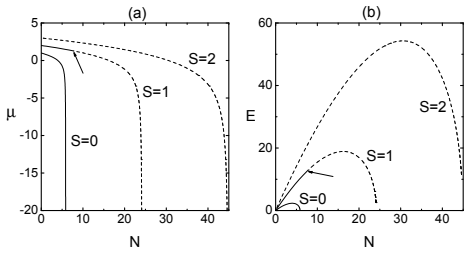

FIG. 5: The chemical potential $\mu$ (a) and the energy $E$ (b) versus norm $N$ for the 2 D trapped modes with vorticities $S=0,1$, and 2, as per Ref. 21]. Solid and dashed lines denote stable and unstable solutions. The arrow indicates the point where the vortices $S=1$ loose their stability.

\section{STABILIZATION OF 2D AND 3D SEMI-VORTEX AND MIXED-MODE SOLITONS BY THE SPIN-ORBIT COUPLING}

\section{A. The models}

In this section, in contrast to the previous one, which outlined well-known results, established between 10 and 20 years ago [18, 21, 22], very recent findings are summarized, which put forward a completely new approach to the stabilization of vorticity-carrying $2 \mathrm{D}$ and $3 \mathrm{D}$ solitons in free space, i.e., without the use of any external potential. The novel stabilization mechanism can be realized in recently introduced models of binary (pseudo-spinor) atomic BEC with the SOC.

A great deal of attention has been lately drawn to the use of ultracold quantum gases, both bosonic and fermionic, as simulators of various fundamental effects that were previously predicted and/or discovered experimentally in much more complex settings of condensed-matter physics [39]. In particular, much interest has been recently attracted to the implementation of the (pseudo-) SOC in atomic BEC, as an efficient emulation of the fundamental SOC and Zeeman-splitting effects in semiconductors, where SOC is induced by the coupling of the electron's magnetic moment to the magnetic field generated by the intrinsic electrostatic field of the crystal in the reference frame moving along with the electron [40, 41]. The experimental implementation of the pseudo-SOC was proposed [42] and realized in 


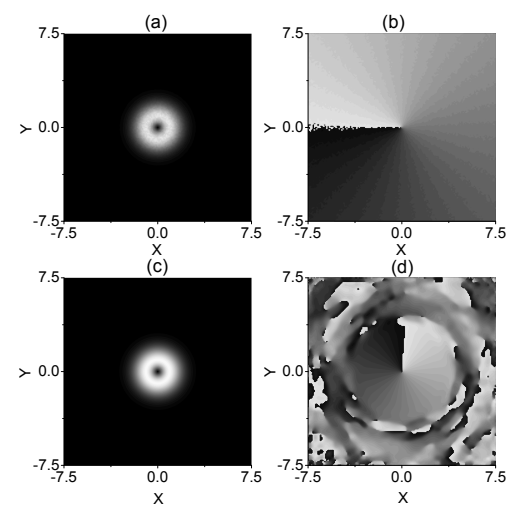

FIG. 6: Grey-scale plots illustrating recovery of a perturbed 2D trapped vortex mode with $S=1$ for $\mu=1.4$ and $N=6.64$, which belongs to stability interval (19). (a,b) Intensity and phase fields in the initial vortex, contaminated by a random-noise perturbation. (c,d) The same in the self-cleaned vortex mode at $t=120$, as per Ref. [21].

the condensate of ${ }^{87} \mathrm{Rb}$ atoms, using appropriately designed laser illumination and magnetic fields [43]. Parallel to that, a large number of theoretical studies on this topic have been carried out [44]-[49], [23, 24]. Reviews of this field are available too [50]. The SOC emulation in the atomic condensate is provided by mapping the spinor wave function of semiconductor electrons onto a two-component pseudo-spinor wave function of the binary BEC composed of atoms in two different hyperfine states. Namely, a pair of states of the ${ }^{87} \mathrm{Rb}$ atom, $\left|\psi_{+}\right\rangle=\left|F=1, m_{F}=0\right\rangle$ and $\left|\psi_{-}\right\rangle=\left|F=1, m_{F}=-1\right\rangle$, were used to map the spin-up and spin-down electron's wave function into them [43]. Thus, the dynamics of the fermionic wave functions of electrons in the semiconductor can be mapped into the mean-field dynamics of the bosonic gas.

The consideration of the interplay of the SOC, which is, essentially, a linear mixing between the two components of the spatially inhomogeneous binary BEC, and the intrinsic nonlinearity in the bosonic condensate has made it possible to predict diverse nonlinear patterns strongly affected or created by the SOC, including a variety of 1D solitons [45], 2D gap solitons supported by optical-lattice potentials [46], and 2D vortices and vortex lattices, in forms specific to the SO-coupled BEC [47].

Here, we will consider the 2D model of the binary SOC BEC based on the following system of based on the following 


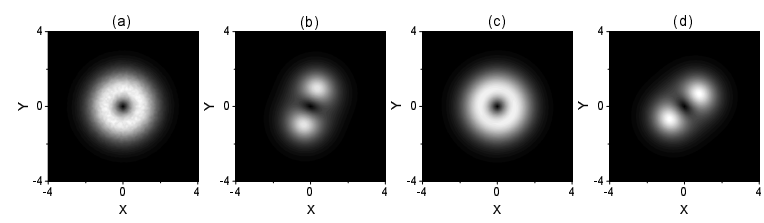

FIG. 7: Periodic evolution of the unstable vortex with $S=1, \mu=1.2$ and $N=8.48$, which belongs to interval (20) of the semistability, as per Ref. 21] In this dynamical regime, the trapped mode periodically splits into two fragments and recombines, while its vorticity is conserved: (a) $t=0$, (b) $t=100$, (c) $t=140$, and (d) $t=180$.

system of the coupled GPEs for up and down components of the spinor wave function, $\Psi \equiv\left\{\psi_{+}, \psi_{-}\right\}[23]$ :

$$
\begin{gathered}
{\left[i \frac{\partial}{\partial t}+\frac{1}{2} \nabla^{2}+i \lambda\left(-\sigma_{y} \frac{\partial}{\partial x}+\sigma_{x} \frac{\partial}{\partial y}+\Omega \sigma_{z}\right)\right.} \\
\left.+\left(\begin{array}{cc}
\left|\psi_{+}\right|^{2}+\eta\left|\psi_{-}\right|^{2} & 0 \\
0 & \left|\psi_{-}\right|^{2}+\eta\left|\psi_{+}\right|^{2}
\end{array}\right)\right]\left(\begin{array}{c}
\psi_{+} \\
\psi_{-}
\end{array}\right)=0,
\end{gathered}
$$

where $\nabla^{2}=\partial^{2} / \partial x^{2}+\partial^{2} / \partial y^{2}, \sigma_{x, y, z}$ are the Pauli matrices, $\lambda$ is a real coefficient of the SOC of the Rashba type (the Dresselhaus coupling, with combination $\sigma_{x} \partial / \partial x-\sigma_{y} \partial / \partial y$, instead of $-\sigma_{u} \partial / \partial x+\sigma_{x} \partial / \partial y$ in Eq. (22), is not considered here, as it tends to destroy $2 \mathrm{D}$ solitons, rather than to create them [51]), $\eta$ is the relative strength of the cross-attraction between the components (cf. Eq. (21a)), under the condition that the strength of the self-attraction is normalized to be 1 , and $\Omega$ is the strength of the Zeeman splitting. Coefficient $1 / \lambda$ has the dimension of length, defining a fixed scale which breaks the above-mentioned scale invariance of the NLSE/GPE in the free 2D space. This effect makes it possible to create stable solitons with norms falling below the collapse threshold, see below.

Stationary solutions of Eq. (22) for 2D solitons with real chemical potential $\mu$ are looked for as $\psi_{ \pm}=$ 
$\exp (-i \mu t) u_{ \pm}(x, y)$, where complex stationary wave functions are determined by equations

$$
\begin{aligned}
\mu u_{+}= & -\frac{1}{2} \nabla^{2} u_{+}-\left(\left|u_{+}\right|^{2}+\gamma\left|u_{-}\right|^{2}\right) u_{+}+ \\
& \left(\frac{\partial u_{-}}{\partial x}-i \frac{\partial u_{-}}{\partial y}\right)-\Omega u_{+}, \\
\mu u_{-}= & -\frac{1}{2} \nabla^{2} u_{-}-\left(\left|u_{-}\right|^{2}+\gamma\left|u_{+}\right|^{2}\right) u_{-} \\
& \left(\frac{\partial u_{+}}{\partial x}+i \frac{\partial u_{+}}{\partial y}\right)+\Omega u_{-} .
\end{aligned}
$$

Dynamical invariants of Eqs. (22) are the total norm, energy, and linear momentum:

$$
\begin{gathered}
N=\iint\left(\left|\psi_{+}\right|^{2}+\left|\psi_{-}\right|^{2}\right) d x d y \equiv N_{+}+N_{-} \\
E=\iint\left\{\frac{1}{2}\left(\left|\nabla \psi_{+}\right|^{2}+\left|\nabla \psi_{-}\right|^{2}\right)-\frac{1}{2}\left(\left|\psi_{+}\right|^{4}+\left|\psi_{-}\right|^{4}\right)-\gamma\left|\psi_{+}\right|^{2}\left|\psi_{-}\right|^{2}+\Omega\left(\left|\psi_{-}\right|^{2}\left|\psi_{+}\right|^{2}\right)\right. \\
\left.+\lambda\left[\psi_{+}^{*}\left(\frac{\partial \psi_{-}}{\partial x}-i \frac{\partial \psi_{-}}{\partial y}\right)+\psi_{-}^{*}\left(-\frac{\partial \psi_{+}}{\partial x}-i \frac{\partial \psi_{+}}{\partial y}\right)\right]\right\} d x d y \\
\mathbf{P}=i \iint\left(\psi_{+}^{*} \nabla \psi_{+}+\psi_{-}^{*} \nabla \psi_{-}\right) d x d y .
\end{gathered}
$$

It is relevant to note that a solution to the linearized version of Eq. (22), in the form of $\psi_{ \pm}(\mathbf{r}, t)=$ $\psi_{ \pm}^{(0)} \exp (i \mathbf{k} \cdot \mathbf{r}-i \omega t)$, gives rise to two branches of the spectrum,

$$
\omega=\left\{\frac{k^{2}}{2}+\sqrt{\lambda^{2} k^{2}+\Omega^{2}}, \frac{k^{2}}{2}-\sqrt{\lambda^{2} k^{2}+\Omega^{2}}\right\},
$$

which is gapless in the case of $\Omega=0$, and features a gap, $0 \leq \omega \leq \Omega$, if the Zeeman splitting is included.

The consistent derivation of the effective 2D SOC model from the full 3D system of GPEs may give rise to the 2D equations with nonpolynomial nonlinearity, as a generalization of the cubic terms in Eq. (22) [48]. This generalized system also creates stable solitons in the 2D free space. Another relevant generalization addresses a model of a dualcore nonlinear coupler in optics, where the SOC is emulated by temporal dispersion of the linear inter-core coupling [53]. It was demonstrated that this model gives rise to stable 2D spatiotemporal solitons ("light bullets").

The 3D model of the SOC system will be taken as per Ref. [24]:

$$
\begin{gathered}
{\left[i \frac{\partial}{\partial t}+\frac{1}{2} \nabla^{2}+i \lambda \nabla \cdot \boldsymbol{\sigma}\right.} \\
\left.+\left(\begin{array}{cc}
\left|\psi_{+}\right|^{2}+\eta\left|\psi_{-}\right|^{2} & 0 \\
0 & \left|\psi_{-}\right|^{2}+\eta\left|\psi_{+}\right|^{2}
\end{array}\right)\right]\left(\begin{array}{c}
\psi_{+} \\
\psi_{-}
\end{array}\right)=0
\end{gathered}
$$

where $\lambda$ is again the SOC coefficient, and the $3 \mathrm{D}$ matrix vector is $\boldsymbol{\sigma}=\left\{\sigma_{x}, \sigma_{y}, \sigma_{z}\right\}$. The 3D system conserves the norm and momentum, given by $3 \mathrm{D}$ counterparts of Eqs. (25) and (27), and energy

$$
\begin{gathered}
E_{\mathrm{tot}}=E_{\mathrm{kin}}+E_{\mathrm{int}}+E_{\mathrm{SOC}}, \\
E_{\mathrm{kin}}=\frac{1}{2} \iiint\left(\left|\nabla \psi_{+}\right|^{2}+\left|\nabla \psi_{-}\right|^{2}\right) d x d y d z, \\
E_{\mathrm{int}}=-\frac{1}{2} \iiint\left(\left|\psi_{+}\right|^{4}+\left|\psi_{-}\right|^{4}+2 \eta\left|\psi_{+} \psi_{-}\right|^{2}\right) d x d y d z \\
E_{\mathrm{SOC}}=-i \lambda \iiint \Psi^{\dagger}(\nabla \cdot \boldsymbol{\sigma}) \Psi d x d y d z .
\end{gathered}
$$

The Zeeman splitting is not included into the 3D system, but it may be added to it. 


\section{B. Stable 2D solitons: standing and mobile semi-vortices and mixed modes}

$$
\text { 1. } 2 D \text { semi-vortices (at } \Omega=0 \text { ) }
$$

First, we note that Eq. (22) with $\Omega=0$ (in the absence of the Zeeman splitting) admits stationary solutions with chemical potential $\mu$, written in terms of the polar coordinates:

$$
\psi_{+}(x, y, t)=e^{-i \mu t} f_{1}(\rho), \psi_{-}(x, y, t)=e^{-i \mu t+i \theta} \rho f_{2}(\rho),
$$

where real functions $f_{1,2}(\rho)$ take finite values and have zero derivatives at $\rho=0$, and feature the following asymptotic form at $\rho \rightarrow \infty$ :

$$
f_{1} \approx F \rho^{-1 / 2} e^{-\sqrt{-2 \mu-\lambda^{2}} \rho} \cos (\lambda r+\delta), f_{2} \approx-F r^{-3 / 2} e^{-\sqrt{-2 \mu-\lambda^{2}} \rho} \sin (\lambda r+\delta),
$$

with constants $F$ and $\delta$. As it follows from Eq. (32), the solutions are exponentially localized, as solitons, at

$$
\mu<-\lambda^{2} / 2 \text {. }
$$

Solutions (31) are built as bound states of a fundamental (zero-vorticity, $S_{+}=0$ ) soliton in component $\psi_{+}$and a solitary vortex, with vorticity $S_{-}=1$, in $\psi_{-}$, therefore composite modes of this type are called semi-vortices (SVs) [23]. The invariance of Eq. (11) with respect to transformation

$$
\psi_{ \pm}(r, \theta) \rightarrow \psi_{\mp}(r, \pi-\theta)
$$

gives rise to a semi-vortex which is a mirror image of (31), with $\left(S_{+}=0, S_{-}=1\right)$ replaced by $\left(S_{+}=-1, S_{-}=0\right)$ :

$$
\psi_{+}(x, y, t)=-e^{-i \mu t-i \theta} \rho f_{2}(\rho), \psi_{-}=e^{-i \mu t} f_{1}(\rho) .
$$

Numerically, stable SVs were generated, as solutions to Eq. (10) by means of imaginary-time simulations [52], starting from the Gaussian input,

$$
\psi_{+}^{(0)}=A_{1} \exp \left(-\alpha_{1} \rho^{2}\right), \psi_{-}^{(0)}=A_{2} \rho \exp \left(i \theta-\alpha_{2} \rho^{2}\right),
$$

where $A_{1,2}$ and $\alpha_{1,2}>0$ are real constants. A typical example of the SV is displayed in Fig. 8 (a).

Further, Fig. 8(b) represents the family of the SVs, showing their chemical potential as a function of the norm, cf. Fig. 5(a). Note that the $\mu(N)$ dependence satisfies the VK criterion, $d \mu / d N<0$, which is the above-mentioned necessary condition for the stability of solitary modes supported by the self-attractive nonlinearity. The family of the SV solitons exists precisely in the interval of norms (18), which, as said above, should secure their stability against the critical collapse. It is also worthy to note that there is no finite minimum (threshold) value of $N$ necessary for the existence of the SVs in the free space. In the limit of $\mu \rightarrow-\infty$, the vortex component of the SV vanishes, while the fundamental one degenerates into the usual Townes soliton, with $N=N_{\max }^{(S=0)}$, as shown by means of the dependence of ratio $N_{+} / N$ on $N$ in Fig. 8(d).

Systematic real-time simulations confirm the stability of the whole SV family at $\eta \leq 1$, while they are unstable at $\eta>1$, where, however, there is another family of stable solitons in the form of mixed modes (MMs), see below. In fact, the SVs at $\eta \leq 1$ and MMs at $\eta \geq 1$ are the first ever found examples of stable solitons supported by the cubic self-attractive nonlinearity in the free $2 \mathrm{D}$ space.

The wave form (36) was used not only as the input for the imaginary-time simulations, but also as an ansatz for the application of the VA. The substitution of the ansatz into expression (26) for the energy yields

$$
E_{\mathrm{SV}}=\pi\left[\frac{A_{1}^{2}}{2}-\frac{A_{1}^{4}}{8 \alpha_{1}}+\frac{A_{2}^{2}}{2 \alpha_{2}}-\frac{A_{2}^{4}}{64 \alpha_{2}^{3}}-\frac{\gamma A_{1}^{2} A_{2}^{2}}{4\left(\alpha_{1}+\alpha_{2}\right)^{2}}+\frac{4 \lambda A_{1} A_{2} \alpha_{1}}{\left(\alpha_{1}+\alpha_{2}\right)^{2}}\right],
$$

while norm (25) of the ansatz is $N=\pi\left[A_{1}^{2} /\left(2 \alpha_{1}\right)+A_{2}^{2} /\left(4 \alpha_{2}^{2}\right)\right]$. Then, values of amplitudes $A_{1}, A_{2}$ and inverse squared widths $\alpha_{1}, \alpha_{2}$ of the ansatz are predicted by the minimization of $E$ with respect to the variational parameters, $\partial E_{\text {semi }} / \partial\left(A_{1,2}, \alpha_{1,2}\right)=0$, under the constraint that $N$ is kept constant. Figure 8 (c) displays the comparison of the so predicted amplitude $A_{1}$ and the maximum value of $\left|\psi_{+}(x, y)\right|$ obtained from the numerical solution at $\eta=0$. It is seen that the accuracy of the VA is quite reasonable.

Very recently, the study of 2D SV solitons was extended to a system with long-range anisotropic cubic interactions, mediated by dipole-dipole forces in a bosonic gas composed of atoms carrying magnetic moments [54]. An essentially novel feature found in the nonlocal model is a new parameter of the family of SV solitons, viz., a spontaneous shift of the pivot of the vortical component $\left(\psi_{-}\right)$with respect to its zero-vorticity counterpart $\left(\psi_{+}\right)$. Those solitons also demonstrate an essentially different mobility scenario from the one outlined below for the present model: they respond to an applied kick by drift in the opposite direction (with an effective negative mass) along a spiral trajectory. 


\section{2. $2 D$ mixed modes (at $\Omega=0$ )}

Aside from the SVs, the same SOC system (22) gives rise to another type of vorticity-carrying solitons, in the form of MMs, which combine terms with zero and unitary vorticities, $(S=0, S=-1)$ and $(S=0, S=+1)$, in the spinup and spin-down components, $\psi_{+}$and $\psi_{-}$. Numerically, the MM can be produced by imaginary-time simulations initiated by the following input, which may also serve as the variational ansatz:

$$
\begin{aligned}
& \psi_{+}^{(0)}=A_{1} \exp \left(-\alpha_{1} \rho^{2}\right)-A_{2} \rho \exp \left(-i \theta-\alpha_{2} \rho^{2}\right) \\
& \psi_{-}^{(0)}=A_{1} \exp \left(-\alpha_{1} \rho^{2}\right)+A_{2} \rho \exp \left(i \theta-\alpha_{2} r^{2}\right) .
\end{aligned}
$$

In fact, the MM may be considered as a superposition of the SV (31) and its mirror image (35). Accordingly, symmetry reflection (34) transforms the MM into itself.

A typical example of the MM state is displayed in Fig. 9(a). Note that peak positions of the two components, $\left|\psi_{+}(x, y)\right|$ and $\left|\psi_{-}(x, y)\right|$, in this state are separated along $x$, Fig. 9(d) showing the separation (DX) as a function of the norm. For a small amplitude of the vortex component, $A_{2}$, Eq. (38) yields $\mathrm{DX} \approx A_{2} /\left(\alpha_{1} A_{1}\right)$.

The $\mu(N)$ dependence for the MM family is displayed in Fig. 9(b), which shows that the VK criterion holds in this case too, and, as well as SVs, the MMs do not have any threshold value of $N$ necessary for their existence. The family exists in the interval of $N<\tilde{N}_{\max }^{(S=0)}(\eta)=2 N_{\max }^{(S=0)} /(1+\eta)$, where $N_{\max }^{(S=0)}$ is the critical norm (18) corresponding to the Townes solitons. In the limit of $N \rightarrow \tilde{N}_{\max }^{(S=0)}(\eta)$ the vortex components vanish in the MM, and it degenerates into a two-component Townes soliton, cf. the degeneration of the SV in the limit of $N \rightarrow N_{\max }^{(S=0)}$, which was shown in Figs. 8(b,d). The separation between peaks of the two components vanishes in this limit too, see Fig. 9(d).

The VA for the MM solutions is generated by the insertion of expression (38), as the variational ansatz, into energy functional (26), which yields

$$
E_{\mathrm{MM}}=\pi\left[A_{1}^{2}+\frac{A_{2}^{2}}{\alpha_{2}}-(1+\gamma)\left(\frac{A_{1}^{4}}{4 \alpha_{1}}+\frac{A_{2}^{4}}{32 \alpha_{2}^{3}}\right)-\frac{A_{1}^{2} A_{2}^{2}}{\left(\alpha_{1}+\alpha_{2}\right)^{2}}+\frac{8 \lambda A_{1} A_{2} \alpha_{1}}{\left(\alpha_{1}+\alpha_{2}\right)^{2}}\right],
$$

the total norm of the ansatz being $N=\pi\left[A_{1}^{2} / \alpha_{1}+A_{2}^{2} /\left(2 \alpha_{2}^{2}\right)\right]$. Numerical solution of the respective variational equations, which minimize energy (39) under the constraint of the fixed norm, produces results which are compared in Fig. 9(c) with their numerically found counterparts. The VA for the MM solution is less accurate than for its VA counterpart, cf. Fig. 8(c), but it is usable too.

Direct simulations demonstrate that the MMs are unstable at $\eta<1$, and stable at $\eta \geq 1$, i.e., precisely in the regions where the SVs are, severally, stable and unstable (exactly at $\eta=1$, both the SV and MM solutions are stable [49]). This stability switch between the SV and MM is explained by the comparison of energy (26) for them at equal values of the norm: the energy is smaller for the SV at $\eta<1$, and for the MM at $\eta>1$ [23] (similar to what is shown below in Fig. 13(a) for the 3D system). Accordingly, the SV and MM realize the system's stable GS, respectively, at $\eta<1$ and $\eta>1$, while in the opposite case each soliton species represents an excited state, instead of the GS, being therefore unstable.

(a)

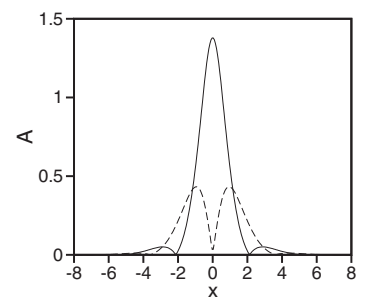

(b)

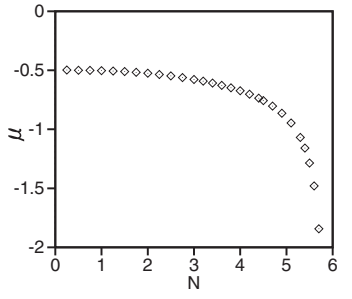

(c)

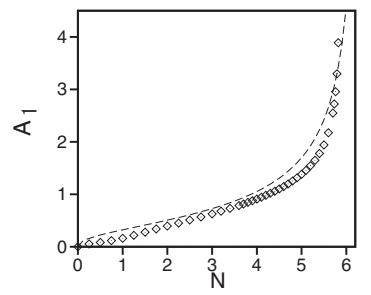

(d)

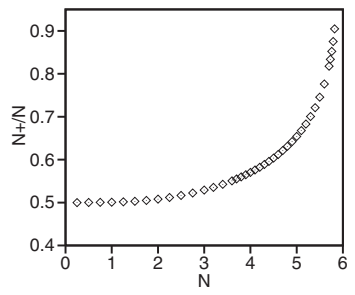

FIG. 8: (a) Cross sections of the fundamental, $\left|\psi_{+}(x, y)\right|$, and vortical, $\left|\psi_{-}(x, y)\right|$, components of a $2 \mathrm{D}$ semi-vortex with $N=5$, along axis $y=0$, are shown by continuous and dashed lines, respectively. (b) Chemical potential $\mu$ vs. norm $N$ for the family of semi-vortices. (c) Comparison of the numerically found amplitude of the semi-vortex solution, $\left|\phi_{+}(x=0, y=0)\right|($ the chain of rhombuses), and its counterpart $A_{1}$, as predicted by the variational approximation based on ansatz (31) (the dashed curve). (d) Ratio $N_{+} / N$ as a function of $N$, for the semi-vortex family. In this figure, $\lambda=1$ and $\eta=\Omega=0$ are fixed in Eq. (22). 
(a)

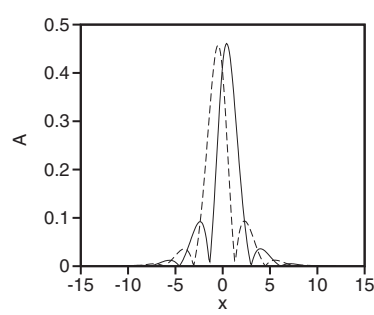

(b)

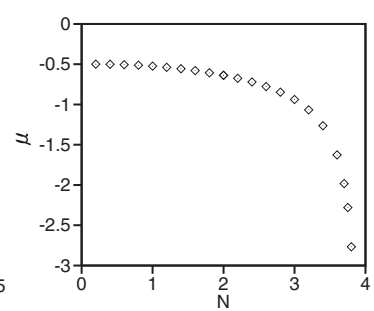

(c)

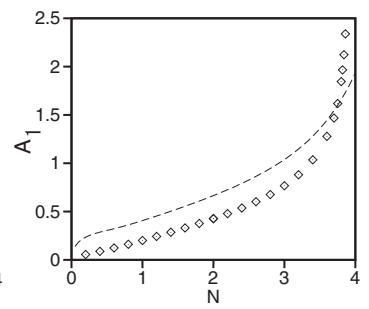

(d)

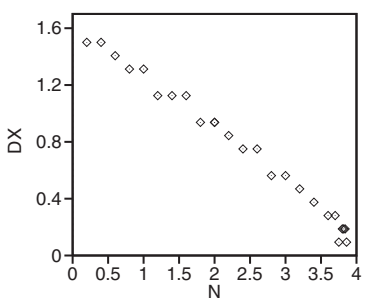

FIG. 9: Panels (a), (b), and (c) display the same as in Fig. 8 but for a $2 \mathrm{D}$ mixed-mode soliton at $\eta=2, \lambda=1$, and $\Omega=0$ in Eq. (22). The norm of the soliton shown in panel (a) is $N=5$. The variational approximation, represented in panel (c), is based on ansatz (38). (d) Separation DX between peak positions of components $\left|\psi_{+}\right|$and $\left|\psi_{-}\right|$vs $N$.

\section{Effects of the Zeeman splitting}

The above considerations did not include the Zeeman splitting, $\Omega \neq 0$. In the presence of moderately strong splitting, with $0<\Omega<\lambda^{2}$, the consideration of Eq. (22), similar to that which produced the asymptotic form (32) of the SV soliton at $\Omega=0$, demonstrates that the SVs exist at

$$
\mu<-(1 / 2)\left(\lambda^{2}+\Omega^{2} / \lambda^{2}\right) \equiv \mu_{\max },
$$

cf. existence region (33) at $\Omega=0$. The asymptotic form of the SV solution, affected by $\Omega$, is more complex than one given by Eq. (32); namely, the radial decay rate and wavenumber in Eq. (32) are replaced as follows:

$$
\sqrt{-2 \mu-\lambda^{2}} \rightarrow \sqrt{-\mu-\lambda^{2}+\sqrt{\mu^{2}-\Omega^{2}}}, \lambda \rightarrow \frac{\sqrt{-2 \mu \lambda^{2}-\lambda^{4}-\Omega^{2}}}{\sqrt{-\mu-\lambda^{2}+\sqrt{\mu^{2}-\Omega^{2}}}} .
$$

Strong Zeeman splitting, with $\Omega>\lambda^{2}$, replaces existence condition (40) by $\mu<-\lambda^{2}$. In this case, the SV solitons keep the asymptotic form (41) in the semi-infinite interval (40) of the chemical potentials, while, in the adjacent finite interval appearing in this case,

$$
\mu_{\max }<\mu<-\lambda^{2},
$$

the SV soliton exhibits a more dramatic change of its asymptotic shape: the radial wavenumber vanishes, the decay of the solution at $\rho \rightarrow \infty$ being provided by the following exponential factor,

$$
\exp \left[-\sqrt{2\left(-\mu-\lambda^{2}-\sqrt{2 \mu \lambda^{2}+\lambda^{4}+\Omega^{2}}\right)} \rho\right]
$$

(at $\mu=\mu_{\max }$, see Eq. (40), expression (43) matches Eq. (410).

As concerns the switch of the stability between the SV and MM at $\eta=1$, which was demonstrated above for $\Omega=0$, it is pushed by $\Omega>0$ to larger values of the relative strength of the cross-attraction, $\eta>1$ [51]. An explicit result can be obtained in the limit of large $\Omega$, when Eq. (23) demonstrates that the chemical potential is close to $-\Omega$ :

$$
\mu=-\Omega+\delta \mu, \quad|\delta \mu| \ll \Omega .
$$

The spin-down component, $\psi_{-}$, is vanishingly small in this limit, hence stationary equation (24) simplifies to

$$
u_{-} \approx \frac{1}{2 \Omega}\left(\lambda \frac{\partial u_{+}}{\partial x}+i \lambda \frac{\partial u_{+}}{\partial y}\right),
$$

where $(\Omega-\mu)$ is approximately replaced by $2 \Omega$, pursuant to Eq. (44). Then, the substitution of approximation (45) into Eq. (23) leads to the following equation for $u_{+}$:

$$
(\delta \mu) u_{+}=-\frac{1}{2}\left(1-\frac{1}{\Omega}\right) \nabla^{2} u_{+}-\left|u_{+}\right|^{2} u_{+} .
$$


By itself, Eq. (46) is tantamount to the stationary version of the 2D NLSE, which gives rise to the Townes solitons in $u_{+}$, while Eq. (45) generates a small vortex component of the SV complex in $u_{-}$. Due to the presence of factor $(1-1 / \Omega)$ in Eq. (46) and the smallness of $1 / \Omega$, the norm (25) of the corresponding SV complex is

$$
N=\left(1-\frac{1}{\Omega}\right) N_{\max }^{(S=0)}+\mathcal{O}\left(\frac{1}{\Omega^{2}}\right),
$$

where $N_{\max }^{(S=0)}$ is the standard norm of the Townes soliton (18), the last term being a contribution from the small vortex component given by Eq. (45). Equation (47) demonstrates that the total norm of the SV soliton is (slightly) smaller than the collapse threshold, $N_{\max }^{(S=0)}$, hence the SV soliton is protected against the collapse, still realizing the stable GS of the system.

The lowest-order approximation presented here does not give rise to terms including the cross-attraction coefficient, $\eta$ from Eq. (22), which implies that, in the limit of large $\Omega$, all solitons belong to the SV type, irrespective of the value of $\eta$, in accordance with the above-mentioned fact that the increase of $\Omega$ leads to conversion of the MMs into SVs [51].

\section{Mobility of stable 2D solitons}

Although the underlying system (22) conserves the momentum (27), the SOC terms break the Galilean invariance of the original NLSEs. For this reason, generating moving solitons from quiescent ones, which were considered above, is a nontrivial problem. As shown in Ref. [23], the system gives rise to the mobility of $2 \mathrm{D}$ solitons along the $y$ axis, but not along $x$. The respective solutions moving at velocity $v_{y}$ can be looked for as

$$
\psi_{ \pm}=\exp \left(i v_{y} y-\frac{i}{2} v_{y}^{2} t\right) \phi_{ \pm}\left(x ; y^{\prime} \equiv y-v_{y} t ; t\right)
$$

(Eq. (48) produces the Galilean transform of the wave functions $\psi_{ \pm}$in Galilean-invariant systems). The substitution of ansatz (48) into Eq. (22) leads to the coupled GPEs in the moving reference frame, which differ from Eqs. (22) by the presence of linear mixing between the two components (here $\nabla^{2} \equiv \partial^{2} / \partial x^{2}+\partial^{2} / \partial\left(y^{\prime}\right)^{2}$, and $\Omega=0$ is set):

$$
\begin{aligned}
& i \frac{\partial \phi_{+}}{\partial t}=-\frac{1}{2} \nabla^{2} \phi_{+}-\left(\left|\phi_{+}\right|^{2}+\gamma\left|\phi_{-}\right|^{2}\right) \phi_{+}^{\prime}+\lambda\left(\frac{\partial \phi_{-}}{\partial x}-i \frac{\partial \phi_{-}}{\partial y^{\prime}}\right)+\lambda v_{y} \phi_{-}, \\
& i \frac{\partial \phi_{-}}{\partial t}=-\frac{1}{2} \nabla^{2} \phi_{-}-\left(\left|\phi_{-}\right|^{2}+\gamma\left|\phi_{+}\right|^{2}\right) \phi_{-}-\lambda\left(\frac{\partial \phi_{+}}{\partial x}+i \frac{\partial \phi_{+}}{\partial y^{\prime}}\right)+\lambda v_{y} \phi_{+} .
\end{aligned}
$$

The same linear mixing can be imposed onto the $2 \mathrm{D}$ settings by a $\mathrm{GHz}$ wave coupling the two underlying atomic states [55], hence the linear mixing by itself represents a relevant addition to the model.

Stationary solutions to equations (49), written in the moving reference frame, can be obtained, as well as in the case of Eqs. (22), by means of the imaginary-time evolution method. In particular, at $\eta=2$, when the GS is represented by the quiescent MM soliton, its moving version, which is displayed in Figs. $8(\mathrm{a}, \mathrm{b})$ for $N=3.1$ and $v_{y}=0.5$, exists and is stable too. As well as its quiescent counterpart, this mode features the mirror symmetry between the profiles of $\left|\phi_{+}(x, y)\right|$ and $\left|\phi_{-}(x, y)\right|$. Figures 10(a,b) display a typical example of a stable moving MM, and Fig. 10(c) shows the amplitude of the moving soliton, $A=\sqrt{\left|\phi_{+}(x=0, y=0)\right|^{2}+\left|\phi_{-}(x=0, y=0)\right|^{2}}$, as a function of $v_{y}$. The amplitude monotonously decreases with the growth of the velocity, vanishing at

$$
v_{y}=\left(v_{y}\right)_{\max }^{(\mathrm{MM})} \approx 1.8
$$

i.e., the mobile solitons exist in the limited interval of the velocities.

The SV solitons may also be made mobile, but in a very narrow interval of velocities - e.g., at $v_{y}<\left(v_{y}\right)_{\max }^{(\mathrm{SV})} \approx 0.03$ for $\eta=0, \lambda=1, \Omega=0$, and $N=3.7$. At $v_{y}>0.03$, the imaginary-time solution of Eq. (49) with the SV input converges to stable MM solitons, instead of the SV.

\section{3D metastable solitons}

\section{Analytical considerations}

The creation of metastable 3D solitons in the model based on Eq. (29) can be predicted starting from scaling evaluation of different terms in the respective energy functional (30). Assuming that a localized state has characteristic 
(a)

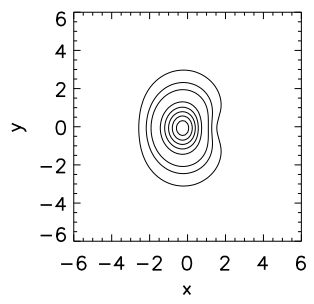

(b)

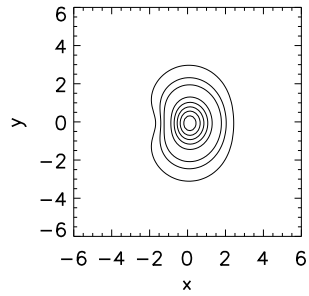

(c)

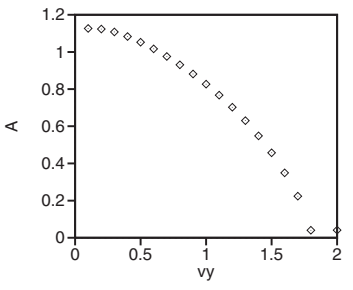

FIG. 10: Contour plots of $\left|\psi_{+}(x, y)\right|$ (a) and $\left|\psi_{-}(x, y)\right|$ (b) of the 2D stable mixed-mode soliton with norm $N=3.1$, moving at velocity $v_{y}=0.5$, for $\eta=2, \lambda=1, \Omega=0$. (c) The amplitude of the moving solitons as a function of $v_{y}$.

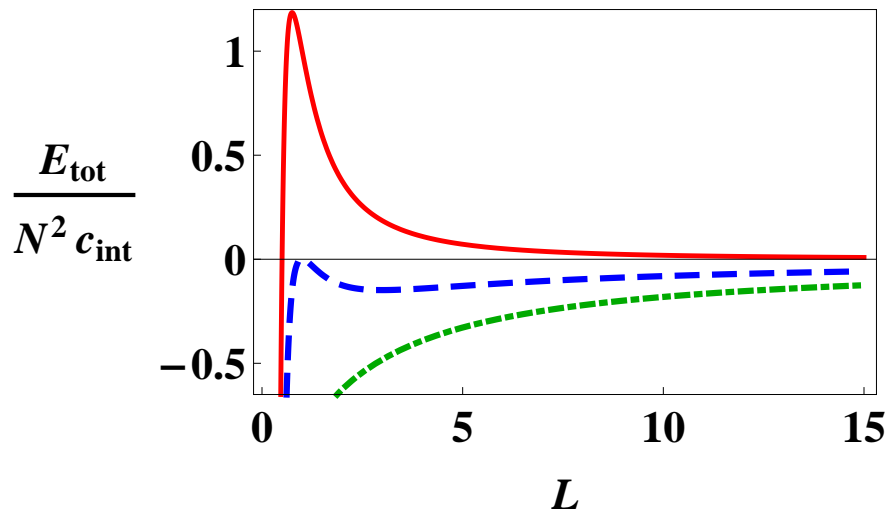

FIG. 11: (Color online) $E_{\text {tot }}$ as a function of condensate's size $L$, as per Eq. (51). The red solid, blue dashed, and green dot-dashed lines represent the energy's variation when $\lambda=0$, and $\lambda>0$ does or does not satisfy condition (52), respectively.

size $L$ and norm $N$, an estimate for the amplitudes of the wave function is $A \sim \sqrt{N} L^{-3 / 2}$. Accordingly, the three terms in Eq. (30) scale with $L$ as

$$
E_{\text {tot }} / N \sim c_{\text {kin }} L^{-2}-c_{\text {soc }} \lambda L^{-1}-\left(c_{\text {int }}^{(\text {self })}+c_{\text {int }}^{(\text {cross })} \eta\right) N L^{-3},
$$

with positive coefficients $c_{\text {kin }}, c_{\text {soc }}$, and $c_{\text {int }}^{\text {(self/cross) }}$. As shown in Fig. 11. Eq. (51) gives rise to a local minimum of $E_{\text {tot }}(L)$ at finite $L$, provided that

$$
0<\lambda N<c_{\mathrm{kin}}^{2} /\left[3\left(c_{\mathrm{int}}^{(\mathrm{self})}+c_{\mathrm{int}}^{(\mathrm{cross})} \eta\right) c_{\mathrm{soc}}\right]
$$

Although this minimum cannot represent the GS, which formally corresponds to $E_{\text {tot }} \rightarrow-\infty$ at $L \rightarrow 0$ in the collapsed state, as is suggested by Fig. 11] too - in fact, this means that the system has no true GS), it corresponds to a selftrapped state which is stable against small perturbations. Condition (52) suggests that the metastable 3D solitons may exist when the SOC term is present, while its strength $\lambda$ is not too large, $N$ and $\eta$ being not too large either.

More accurate semi-analytical results can be obtained by means of the VA, which is based on the following ansatz for the 3D version of the SV mode, written in cylindrical coordinates $(\rho, z, \theta)$, cf. its 2D counterpart (36):

$$
\begin{gathered}
\left\{\psi_{+}, \psi_{-}\right\}=e^{-i \mu t}\left\{f_{1}(\rho, z), e^{i \theta} f_{2}(\rho, z)\right\}, \\
f_{n}=i^{n-1}\left(A_{n}+i B_{n} z\right) \rho^{n-1} \exp \left(-\alpha_{n} \rho^{2}-\beta_{n} z^{2}\right) \quad(n=1,2),
\end{gathered}
$$

with real parameters $A_{n}, B_{n}$, and $\alpha_{n}>0, \beta_{n}>0$. In particular, amplitudes $A_{n}$ and $B_{n}$ account for possibility of having even and odd terms in the dependence of the wave functions of the vertical coordinate, $z$. Further, the ansatz for the 3D version of the MM soliton may be chosen as a superposition, with mixing angle $\zeta$, of the SV (53) and its flipped counterpart, cf. Eq. (35) in the 2D setting:

$$
\begin{aligned}
& \psi_{+}=(\cos \zeta) f_{1}(\rho, z)-(\sin \zeta) f_{2}^{*}(\rho, z) e^{-i \phi} \\
& \psi_{-}=(\sin \zeta) f_{1}^{*}(\rho, z)+(\cos \zeta) f_{2}(\rho, z) e^{i \phi}
\end{aligned}
$$


The substitution of ansätze (53)-(55) into the full energy (30) and minimizing it with respect to the free parameters under the constraint of the fixed total norm produces results summarized in Fig. 12, in which (meta)stable 3D solitons are predicted to exist in the shaded areas. It is seen that the solitons indeed exist, provided that $\lambda, N$ and $\eta$ are not too large, in agreement with the rough prediction of Eq. (52). In other words, for fixed $\lambda$ and $\eta$ the stable 3D solitons exist in a finite interval of the norm,

$$
0 \leq N \leq N_{\max }(\lambda, \eta)
$$

which is qualitatively similar to the situation for the single-component model stabilized by the HO trapping potential, cf. Figs. [1] and 2,
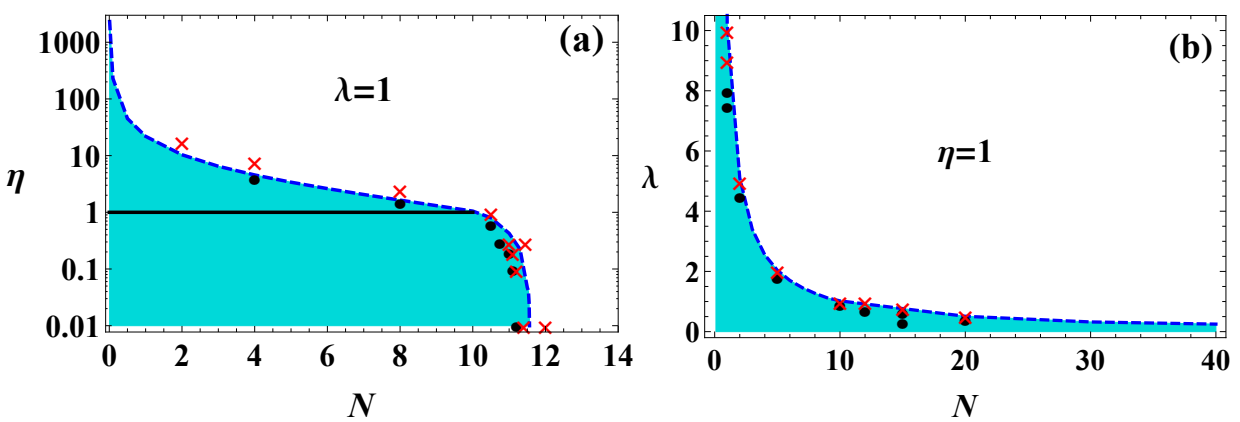

FIG. 12: (Color online) Stable 3D solitons are predicted by the variational approximation in blue shaded regions of the parameter planes. In panel (a), these are semi-vortices at $\eta<1$, and mixed modes at $\eta>1$, with the boundary between them depicted by the black solid line. In (b), the stability area is filled by the solitons of both types, as they have equal energies at $\eta=1$. The predictions are confirmed by numerical findings, as indicated by red crosses and black dots, which indicate, respectively, the absence and presence of stable numerically generated 3D solitons for respective sets of parameters.

As shown in Fig. 13(a), for $\eta<1$ the VA-predicted energy of the SV is lower than that for the MM, and vice versa for $\eta>1$, similar to what was found in the 2D model (22) with $\Omega=0$. Furthermore, red squares in Fig. 13(b) depict the VA prediction for the soliton's chemical potential, $\mu$, plotted as a function of norm $N$ for $\lambda=1$ and $\eta=0.3$. These curves clearly demonstrate that, also similar to what was found in the $2 \mathrm{D}$ model (cf. Figs. 8(b) and 9(b)), there is no threshold (minimum norm) necessary for the existence of the stable $3 \mathrm{D}$ solitons, which exist up to a $N=N_{\max }$, see Eq. (56).
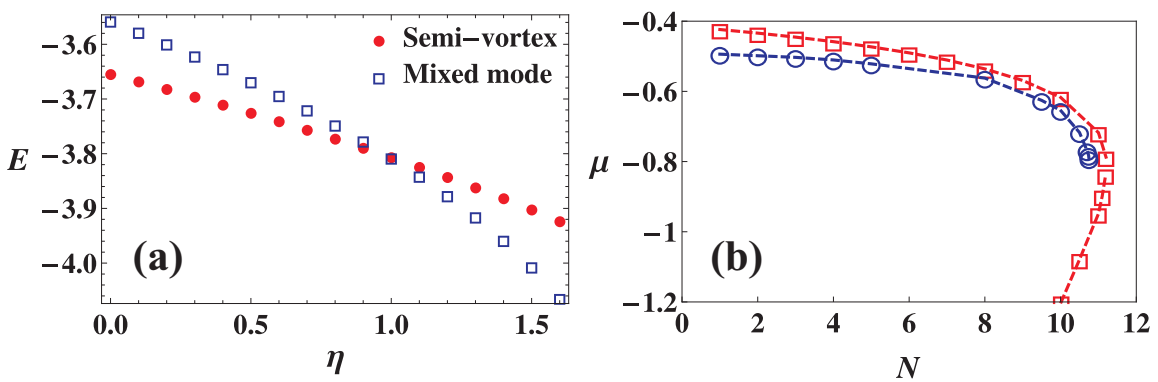

FIG. 13: (Color online) (a) Energies of the semi-vortices and mixed modes versus the cross-attraction strenght, $\eta$, as predicted by the variational approximation for $\lambda=1$ and $N=8$ (as per Ref. [24]). The two curves cross at $\eta=1$, where the energies are equal. (b) Numerically (blue circles) and variationally (red squares) found chemical potentials versus the norm for the semi-vortices at $\lambda=1$ and $\eta=0.3$.

While the stability of the upper branch is consistent with the VK criterion, $d \mu / d N<0$, the lower branch in Fig. 13(b), which does not satisfy the VK criterion, represents solitons corresponding to the energy maximum (instead of the minimum) of the blue dashed curve in Fig. 11, therefore the lower-branch solitons are definitely unstable. In the limit of $\mu \rightarrow-\infty$, they carry over into the known unstable 3D solitons of the single GPE [56]. 


\section{Numerical results}

In the numerical form, stationary 3D solitons were produced by means of imaginary-time simulations of Eq. (29). Typical examples of the density profiles in stable SV and MM solitons are displayed in Fig. 14 Naturally, the MM states exhibit a more sophisticated profile.

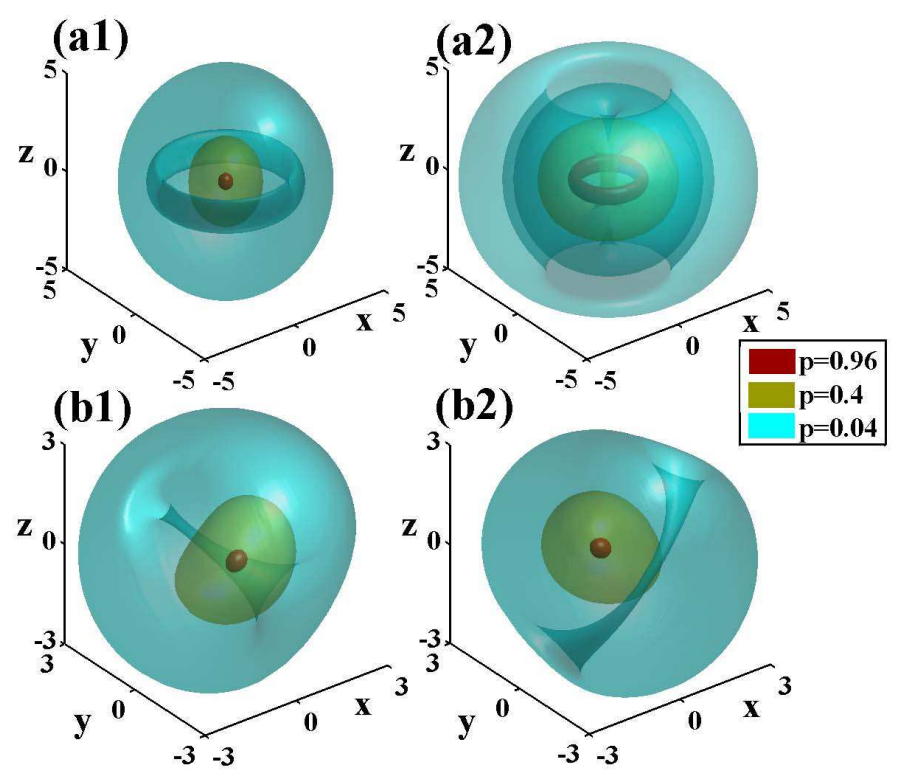

FIG. 14: (Color online) Density profiles of 3D solitons for $N=8$ and $\lambda=1$, as per Ref. [24]. (a) A semi-vortex for $\eta=0.3$, whose fundamental and vortical components, $\left|\psi_{+}\right|$and $\left|\psi_{-}\right|$, are plotted in (a1) and (a2), respectively. (b) A mixed mode for $\eta=1.5$, with (b1), (b2) displaying $\left|\psi_{+}\right|$and $\left|\psi_{-}\right|$, respectively. In each subplot, different colors represent constant-magnitude surfaces, $\left|\psi_{ \pm}(x, y, z)\right|=(0.96,0.4,0.04) \times\left|\psi_{ \pm}\right| \max$.

Symbols in Fig. 12, which indicate the absence and presence of the numerically generated stable solitons, are in good agreement with the predictions of the VA. Further, blue circles in Fig. 13.(b) represent the numerically obtained soliton's chemical potential, which is also close to the prediction of the VA. However, the unstable (lower) branch in Fig. 13(b), predicted by the VA, cannot be produced by the imaginary-time integration. The stability of the solitons belonging to the upper branch in Fig. 13(b) was verified by real-time simulations of their perturbed evolution. Further, similar to what is shown above for the 2D system in Fig. 11, it was found that stable 3D solitons may be set in motion in a limited interval of velocities, cf. Eq. (50).

Lastly, it was mentioned in Section II that all the higher-order vortex modes, with $S \geq 2$, are completely unstable in the model with the HO trapping potential (see Figs. 3 and 5). Higher-vorticity states of both the SV and MM types can be found too as stationary solutions of Eqs. (22) 23] and 29] 24], starting from ansätze

$$
\psi_{+}^{(0)}=A_{1} \rho^{s} \exp \left(i s \theta-\alpha_{1} \rho^{2}\right), \psi_{-}^{(0)}=A_{2} \rho^{s+1} \exp \left(i(s+1) \theta-\alpha_{2} \rho^{2}\right),
$$

with $s=1,2,3, \ldots$, for the higher-order 2D SV solitons (cf. Eq. (36)), and

$$
\begin{gathered}
\left\{\psi_{+}, \psi_{-}\right\}=e^{-i \mu t+i s \theta}\left\{f_{1}(\rho, z), e^{i \theta} f_{2}(\rho, z)\right\}, \\
f_{n}=i^{n-1}\left(A_{n}+i B_{n} z\right) \rho^{s+n-1} \exp \left(-\alpha_{n} \rho^{2}-\beta_{n} z^{2}\right) \quad(n=1,2),
\end{gathered}
$$

for their 3D counterparts, cf. Eq. (53) . However, numerical analysis demonstrates that all such higher-order states, with $s \geq 1$, are completely unstable, in the $2 \mathrm{D}$ and $3 \mathrm{D}$ systems alike.

\section{CONCLUSION}

This article aims to present a brief review of the broad area of 2D and 3D solitons, both fundamental (zero-vorticity) and vortical ones. The multidimensional solitons and solitary vortices find most important physical realizations in 
BEC and nonlinear optics, the most essential problem being the search for physically relevant settings which provide for the stabilization of this states against the collapse and splitting, that tend to destroy the fundamental and vortical solitons, respectively, in media with the (most common) cubic self-attractive nonlinearity. The presentation includes two particular topics, one well-established, viz., the stabilization of the 3D and 2D modes with vorticities $S=0$ and 1 in a trapping harmonic-oscillator potential (generally, anisotropic one), and the most recent topic, namely, the creation of stable $2 \mathrm{D}$ and $3 \mathrm{D}$ solitons, which mix terms with $S=0$ and 1 (semi-vortices) or $S=0$ and \pm 1 (mixed modes), in a two-component system which realizes the spin-orbit coupling in BEC. In either case, the analysis reveals a drastic difference between the 2D and 3D settings. In the former case, the stabilization mechanism creates GSs (ground states), that were missing without it. The total norm of the GSs takes values below the threshold necessary for the onset of the critical collapse, driven by the cubic attractive nonlinearity in the $2 \mathrm{D}$ space. In the $3 \mathrm{D}$ settings, the supercritical collapse does not allow the creation of a GS, but, nevertheless, an appropriate mechanism may create metastable solitons, which are robust against small perturbations.

There are many directions for the development of the studies of multidimensional solitons. As concerns the theory, a completely different possibility is the use of spatially nonuniform self-repulsive nonlinearity, with the local strength growing from the center to periphery at any rate faster than $r^{D}$, where $r$ is the distance from the center, and $D$ the spatial dimension. This model makes it possible to create very robust solitons of diverse types, including fundamental ones and solitary vortices [57, 58], as well as sophisticated 3D states - notably, hopfions (vortex tori with inner twist, which feature two independent topological numbers). A challenging problem is finding still more general physically relevant conditions for the creation of complex 3D modes, such as the hopfions 60], skyrmions (which, similar to hopfions, carry two different topological charges) [61, 62], monopoles [63], linked vortex rings, and others. In terms of the experiment, the entire area of multidimensional solitons remains a challenging one, as very few experimental results have been reported, thus far (the latest experimental findings are the creation of $(2+1) \mathrm{D}$ optical spatial solitons in media with competing focusing-defocusing cubic-quintic 64] and quintic-septimal [65] nonlinearities, as well as the making of a quasi-stable vortex solitons in an optical medium where nonlinear losses are essential [66]).

\section{Acknowledgments}

I would like to thank my collaborators in original works on various topics related to multidimensional solitons (most of those works are cited in this review): F. Kh. Abdullaev, C. B. de Araújo, B. B. Baizakov, V. Besse, O. V. Borovkova, G. Boudebs, C. M. Brtka, W. B. Cardoso, R. Carretero-González, Z. Chen, J. Cuevas, R. Driben, N. Dror, P. Dyke, Z. Fan, D. J. Frantzeskakis, A. Gammal, G. Gligorić, L. Hadžievski, R. G. Hulet, X. Jiang, Y. V. Kartashov, P. G. Kevrekidis, V. V. Konotop, H. Leblond, B. Li, Y. Li, V. E. Lobanov, D. Luo, A. Maluckov, D. Mazilu, T. Meier, D. Mihalache, J. H. V. Nguyen, W. Pang, H. Pu, A. S. Reyna, H. Sakaguchi, L. Salasnich, M. Salerno, E. Ya. Sherman, Ya. Shnir, H. Susanto, F. Toigo, L. Torner, F. Wise, Y.-C. Zhang, and Z.-W. Zhou. I also thank Carlos Pando-Lambruschini for the invitation to submit the paper to the special issue of the European Physical Journal Special Topics.

Parts of this work were supported by grants No. I-1024-2.7/2009 from the German-Israel Foundation, and No. 2010239 from the Binational (US-Israel) Science Foundation.

[1] Yu. S. Kivshar and D. E. Pelinovsky, Phys. Rep. 331, 117 (2000); B. A. Malomed, D. Mihalache, F. Wise, and L. Torner, J. Optics B: Quant. Semicl. Opt. 7, R53-R72 (2005); A. S. Desyatnikov, L. Torner, and Y. S. Kivshar, Progr. Opt. 47, 1 (2005); D. Mihalache, Rom. J. Phys. 57, 352-371 (2012).

[2] Y. S. Kivshar and G. P. Agrawal, Optical Solitons: From Fibers to Photonic Crystals (Academic Press, San Diego, 2003);

[3] Y. V. Kartashov, B. A. Malomed, and L. Torner, Rev. Mod. Phys. 83, 247(2011).

[4] N. R. Cooper, Phys. Rev. Lett. 82, 1554 (1999).

[5] E. Babaev, Phys. Rev. Lett. 88, 177002 (2002).

[6] N. Kumada, A. Sawada, Z. F. Ezawa, S. Nagahama, H. Azuhata, K. Muraki, T. Saku, and Y. Hirayama, J. Phys. Soc. Jpn. 69, 3178 (2000); W. Munzer, A. Neubauer, T. Adams, S. Muhlbauer, C. Franz, F. Jonietz, R. Georgii, P. Böni, B. Pedersen, M. Schmidt, A. Rosch, and C. Pfleiderer, Phys. Rev. B 81, 041203 (2010).

[7] R. Alkofer, H. Reinhardt, and H. Weigel, Phys. Rep. 265, 139 (1996); M. Bender, P. H. Heenen, and P. G. Reinhard, Rev. Mod. Phys. 75, 121 (2003).

[8] H. Aratyn, L. A. Ferreira, and A. H. Zimerman, Phys. Rev. Lett. 83, 1723 (1999); P. Sutcliffe, Proc. R. Soc. A 463, 3001 (2007).

[9] B. Kleihaus, J. Kunz, and Y. Shnir, Phys. Rev. D 68, 101701 (2003); J. Kunz, U. Neemann, and Y. Shnir, Phys. Lett. B 640, 57 (2006); E. Radu and M. S. Volkov, Phys. Rep. 468, 101 (2008).

[10] A. Hosaka and H. Toki, Phys. Rep. 227, 65-188 (1996); T. Sakai and S. Sugimoto, Prog. Theor. Phys. 113, 843(2005). 
[11] Y. Silberberg, Opt. Lett. 15, $1282(1990)$.

[12] R. McLeod, K. Wagner, and S. Blair, Phys. Rev. A 52, 3254 (1995).

[13] A. D. Martin and J. Ruostekoski, New J. Phys. 14, 043040 (2012); J. Cuevas, P. G. Kevrekidis, B. A. Malomed, P. Dyke, and R. G. Hulet, New J. Phys. 15, 063006 (2013); J. H. V. Nguyen, P. Dyke, D. Luo, B. A. Malomed, and R. G. Hulet, Nature Phys. 10, 918-922 (2014); G. D. McDonald, C. C. N. Kuhn, K. S. Hardman, S. Bennetts, P. J. Everitt, P. A. Altin, J. E. Debs, J. D. Close, and N. P. Robins, Phys. Rev. Lett. 113, 013002 (2014); H. Sakaguchi and B. A. Malomed, New J. Phys., in press.

[14] T. Dauxois and M. Peyrard, Physics of Solitons (Cambridge University Press: Cambridge, UK, 2006).

[15] L. Bergé, Phys. Rep. 303, 259(1998); C. Sulem and P. L. Sulem, The nonlinear Schrödinger equation: self-focusing and wave collapse (Springer: Berlin, 1999); E. A. Kuznetsov and F. Dias, Phys. Rep. 507, 43(2011); G. Fibich, The Nonlinear Schrödinger Equation: Singular Solutions and Optical Collapse (Springer: Heidelberg, 2015).

[16] R. Y. Chiao, E. Garmire, and C. H. Townes, Phys. Rev. Lett. 13, 479 (1964).

[17] V. I. Kruglov, Yu. A. Logvin, and V. M. Volkov, J. Mod. Opt. 39, 2277(1992).

[18] F. Dalfovo and S. Stringari, Phys. Rev. A 53, 2477 (1996); R. J. Dodd, J. Res. Natl. Inst. Stand. Technol. 101, 545 (1996); T. J. Alexander and L. Bergé, Phys. Rev. E 65, 026611 (2002); L. D. Carr and C. W. Clark, Phys. Rev. Lett. 97, 010403 (2006).

[19] S. K. Adhikari, Phys. Rev. E 65, 016703 (2001).

[20] H. Saito and M. Ueda, Phys. Rev. Lett. 89, 190402 (2002).

[21] D. Mihalache, D. Mazilu, B. A. Malomed, and F. Lederer, Phys. Rev. A 73, 043615 (2006).

[22] B. A. Malomed, F. Lederer, D. Mazilu, and D. Mihalache, Phys. Lett. A 361, 336(2007).

[23] H. Sakaguchi, B. Li, and B. A. Malomed, Phys. Rev. E 89, 032920 (2014).

[24] Y.-C. Zhang, Z.-W. Zhou, B. A. Malomed, and H. Pu, Phys. Rev. Lett. 115, 253902 (2015).

[25] L. P. Pitaevskii and S. Stringari, Bose-Einstein Condensation, Oxford University Press (Oxford, 2003); H. T. C. Stoof, K. B. Gubbels, and D. B. M. Dickerheid, Ultracold Quantum Fields (Springer: Dordrecht, 2009).

[26] B. B. Baizakov, B. A. Malomed and M. Salerno, Phys. Rev. A 70, 053613 (2004); D. Mihalache, D. Mazilu, F. Lederer, Y. V. Kartashov, L.-C. Crasovan, and L. Torner, Phys. Rev. E 70, 055603(R) (2004); H. Leblond, B. A. Malomed, and D. Mihalache, Phys. Rev. E 76, 026604 (2007).

[27] L. Chomaz, L. Corman, T. Bienaime, R. Desbuquois, C. Weitenberg, S. Nascimbène, J. Beugnon, and J. Dalibard, Nature Comm. 6, 6162 (2015).

[28] K. E. Strecker, G. B. Partridge, A. G. Truscott, and R. G. Hulet, Nature 417, 150 (2002); L. Khaykovich, F. Schreck, G. Ferrari, T. Bourdel, J. Cubizolles, L. D. Carr, Y. Castin, and C. Salomon, Science 269, 1290 (2002); K. E. Strecker, G. B. Partridge, A. G. Truscott, and R. G. Hulet, New J. Phys. 5, 73.1 (2003).

[29] N. K. Efremidis, S. Sears, D. N. Christodoulides, J. W. Fleischer, and M. Segev, Phys. Rev. E 66, 046602 (2002); B. B. Baizakov, B. A. Malomed, and M. Salerno, Europhys. Lett. 63, 642 (2003); J. Yang and Z. H. Musslimani, Opt. Lett. 28, 2094 (2003).

[30] D. Neshev, T. J. Alexander, E. A. Ostrovskaya, Y. S. Kivshar, H. Martin, I. Makasyuk, and Z. Chen, Phys. Rev. Lett. 92, 123903 (2004); J. W. Fleischer, G. Bartal, O. Cohen, O. Manela, M. Segev, J. Hudock, and D. N. Christodoulides, ibid. 92, 123904 (2004).

[31] E. A. Cerda-Méndez, D. Sarkar, D. N. Krizhanovskii, S. S. Gavrilov, K. Biermann, M. S. Skolnick, and P. V. Santos, Phys. Rev. Lett. 111, 146401 (2013).

[32] B. A. Malomed, Progr. Optics 43, 71; http://www.sciencedirect.com/science/article/pii/S0079663802800269

[33] N. G. Vakhitov and A. A. Kolokolov, Radiophys. Quant. Electron. 16, 783 (1973).

[34] L. Salasnich, B. A. Malomed, and F. Toigo, Phys. Rev. A 76, 063614 (2007).

[35] A. L. Marchant, T. P. Billam, T. P. Wiles, M. M. H. Yu, S. A. Gardiner, and S. L. Cornish, Nature Comm. 4, 1865 (2013); J. Everitt, M. A. Sooriyabandara, G. D. McDonald, K. S. Hardman, C. Quinlivan, P. Manju, P. Wigley, J. E. Debs, J. D. Close, C. C. N. Kuhn, and N. P. Robins, arXiv:1509.06844.

[36] S. L. Cornish, S. T. Thompson, and C. E. Wieman, Phys. Rev. Lett. 96, 170401 (2006).

[37] M. Desaix, D. Anderson, and M. Lisak, J. Opt. Soc. Am. B 8, 2082 (1991).

[38] M. Brtka, A. Gammal, and B. A. Malomed, Phys. Rev. A 82, 053610 (2010).

[39] P. Hauke, F. M. Cucchietti, and L. Tagliacozzo, I. Deutsch, and M. Lewenstein, Rep. Prog. Phys. 75, 082401 (2012); T. H. Johnson, S. R. Clark, and D. Jaksch, EPJ Quantum Technology 1,10 (2014); E. Zohar, J. I. Cirac, and B. Reznik, Rep. Prog. Phys. 79, 014401 (2016).

[40] G. Dresselhaus, Phys. Rev. 100, 580 (1955).

[41] Y. A. Bychkov and E. I. Rashba, J. Phys. C 17, 6039 (1984).

[42] D. L. Campbell, G. Juzeliūnas, and I. B. Spielman, Phys. Rev. A 84, 025602 (2011).

[43] Y. J. Lin, K. Jimenez-Garcia, and I. B. Spielman, Nature 471, 83 (2011); J.-Y. Zhang, S.-C. Ji, Z. Chen, L. Zhang, Z.-D. Du, B. Yan, G.-S. Pan, B. Zhao, Y. J. Deng, H. Zhai, S. Chen, and J.-W. Pan, Phys. Rev. Lett. 109, 115301 (2012); C. Hamner, C. Qu, Y. Zhang, J. Chang, M. Gong, C. Zhang, and P. Engels, Nature Commun. 5, 4023 (2014); A. J. Olson, S.-J. Wang, R. J. Niffenegger, C.-H. Li, C. H. Greene, and Y. P. Chen, Phys. Rev. A 90, 013616 (2014).

[44] Y. Zhang, L. Mao, and C. Zhang, Phys. Rev. Lett. 108, 035302 (2012); Y. Li, L. P. Pitaevskii, and S. Stringari, Phys. Rev. Lett. 108, 225301 (2012); Y. Zhang, G. Chen, and C. Zhang, Scientific Reports 3, 1937 (2013); D. A. Zezyulin, R. Driben, V. V. Konotop, and B. A. Malomed, Phys. Rev. A 88, 013607 (2013); Y.-C. Zhang, Sh.-W. Song, and W.-M. Liu, Scientific Reports 4, 4992 (2014).

[45] V. Achilleos, D. J. Frantzeskakis, P. G. Kevrekidis, and D. E. Pelinovsky, Phys. Rev. Lett. 110, 264101 (2013); Y. V. 
Kartashov, V. V. Konotop, and F. Kh. Abdullaev, ibid. 111, 060402 (2013); Y. Xu, Y. Zhang, and B. Wu, Phys. Rev. A 87, 013614 (2013); L. Salasnich and B. A. Malomed, ibid. 87, 063625 (2013); Y. V. Kartashov, V. V. Konotop, and D. A. Zezyulin, Phys. Rev. A 90, 063621 (2014).

[46] V. E. Lobanov, Y. V. Kartashov, and V. V. Konotop, Phys. Rev. Lett. 112, 180403 (2014).

[47] S. Sinha, R. Nath, and L. Santos, Phys. Rev. Lett. 107, 270401 (2011); C. J. Wu, I. Mondragon-Shem, and X.-F. Zhou, Chin. Phys. Lett. 28, 097102 (2011); Y. Deng, J. Cheng, H. Jing, C. P. Sun, and S. Yi, Phys. Rev. Lett. 108, 125301 (2012); T. Kawakami, T. Mizushima, and K. Machida, Phys. Rev. A 84, 011607 (2011); B. Ramachandhran, B. Opanchuk, X.-J. Liu, H. Pu, P. D. Drummond, and H. Hu, ibid. A 85, 023606 (2012); G. J. Conduit, ibid. 86, 021605(R) (2012); E. Ruokokoski, J. A. M. Huhtamaki, and M. Mottonen, ibid. 86, 051607 (2012); H. Sakaguchi and B. Li, Phys. Rev. A 87, 015602 (2013); A. Fetter, ibid. A 89, 023629 (2014); J. Low Temp. Phys. 180, 37-52 (2015).

[48] L. Salasnich, W. B. Cardoso, and B. A. Malomed, Phys. Rev. A 90, 033629 (2014).

[49] H. Sakaguchi and B. A. Malomed, Phys. Rev. E 90, 062922 (2014).

[50] J. Dalibard, F. Gerbier, G. Juzeliūnas, and P. Öhberg, Rev. Mod. Phys. 83, 1523 (2011); H. Zhai, Int. J. Mod. Phys. B 26, 1230001 (2012); I. B. Spielman, in: Ann. Rev. Cold At. Mol. 1, 145 (2012); V. Galitski and I. B. Spielman, Nature 494, 49 (2013); X. Zhou, Y. Li, Z. Cai, and C. Wu, J. Phys. B: At. Mol. Opt. Phys. 46, 134001 (2013); N. Goldman, G. Juzeliūnas, P. Öhberg, and I. B. Spielman, Rep. Progr. Phys. 77, 126401 (2014).

[51] H. Sakaguchi, E. Ya. Sherman, and B. A. Malomed, Vortex solitons under the Rashba Dresselhaus coupling and Zeeman splitting, to be published.

[52] B. D. Esry, C. H. Greene, J. P. Burke, Jr., and J. L. Bohn, Hartree-Fock theory for double condensates, Phys. Rev. Lett. 78, 3594-3597 (1997); D. L. Feder, M. S. Pindzola, L. A. Collins, B. I. Schneider, and C. W. Clark, Phys. Rev. A 62, 053606 (2000); M. L. Chiofalo, S. Succi, and M. P. Tosi, Phys. Rev. E 62, 7438-7444 (2000); W. Bao and Q. Du, SIAM J. Sci. Comput. 25, 1674-1697 (2004).

[53] Y. V. Kartashov, B. A. Malomed, V. V. Konotop, V. E. Lobanov, and L. Torner, Stabilization of solitons in bulk Kerr media by dispersive coupling, Opt. Lett. 40, 1045-1048 (2015).

[54] X. Jiang, Z. Fan, Z. Chen, W. Pang, Y. Li, and B. A. Malomed, Phys. Rev. A, in press.

[55] H. Saito, R. G. Hulet, and M. Ueda, Phys. Rev. A 76, 053619 (2007); H. Susanto, P. G. Kevrekidis, B. A. Malomed, and F. Kh. Abdullaev, Phys. Lett. A 372, 1631 (2008).

[56] Y. Silberberg, Opt. Lett. 15, 1282 (1990).

[57] O. V. Borovkova, Y. V. Kartashov, B. A. Malomed, and L. Torner, Opt. Lett. 36, 3088-3090 (2011); O. V. Borovkova, Y. V. Kartashov, L. Torner, and B. A. Malomed, Phys. Rev. E 84, 035602 (R) (2011).

[58] Q. Tian, L. Wu, Y. Zhang, and J.-F. Zhang, 85, 056603 (2012); Y. Wu, Q. Xie, H. Zhong, L. Wen, and W. Hai, ibid. A 87, 055801 (2013); R. Driben, Y. V. Kartashov, B. A. Malomed, T. Meier, and L. Torner, Phys. Rev. Lett. 112, 020404 (2014); R. Driben, Y. Kartashov, B. A. Malomed, T. Meier, and L. Torner, New J. Phys. 16, 063035 (2014); R. Driben, N. Dror, B. Malomed, and T. Meier, ibid. 17, 083043 (2015); R. Driben, T. Meier, and B. A. Malomed, Sci. Rep. 5, 9420 (2015); N. Dror and B. A. Malomed, J. Optics 16, 014003 (2016).

[59] Y. V. Kartashov, B. A. Malomed, Y. Shnir, and L. Torner, Phys. Rev. Lett. 113, 264101 (2014).

[60] Y. M. Bidasyuk, A. V. Chumachenko, O. O. Prikhodko, S. I. Vilchinskii, M. Weyrauch, and A. I. Yakimenko, Phys. Rev. A 92, 053603 (2015).

[61] J. Ruostekoski and J. R. Anglin, Phys. Rev. Lett. 86, 3934 (2001); R. A. Battye, N. R. Cooper and P. M. Sutcliffe, ibid. 88, 080401 (2002); C. M. Savage and J. Ruostekoski, ibid. 91, 010403 (20032); J. Ruostekoski and J. R. Anglin, ibid. 91, 190402 (2003).

[62] L. S. Leslie, A. Hansen, K. C. Wright, B. M. Deutsch, and N. P. Bigelow, Phys. Rev. Lett. 103, 250401 (2009); J. Y. Choi, W. J. Kwon, and Y. I. Shin, ibid. 108, 035301 (2012).

[63] M. W. Ray, E. Ruokokoski, S. Kandel, M. Möttönen, and D. S. Hall, Nature 505, 657(2014).

[64] E. L. Falc̃ao-Filho, C. B. de Araújo, G. Boudebs, H. Leblond, and V. Skarka, Phys. Rev. Lett. 110, 013901 (2013).

[65] A. S. Reyna, K. C. Jorge, and C. B. de Araújo, Phys. Rev. A 90, 063835 (2014).

[66] A. S. Reyna, G. Boudebs, B. A. Malomed, and C. B. de Araújo, Phys. Rev. A 93, 013840 (2016). 